\title{
Tomato SIBES1.8 influences leaf morphogenesis by mediating gibberellin metabolism and signaling
}

Deding Su ${ }^{1,2}$, Wei Xiang ${ }^{1,2}$, Qin Liang ${ }^{1,2}$, Ling Wen ${ }^{1,2}$, Yuan Shi ${ }^{1,2}$, Yudong Liu ${ }^{1,2 *}$, Zhiqiang $\mathrm{Xian}^{1,3 *}$ and Zhengguo $\mathrm{Li}^{1,2}$ *

${ }^{1}$ Key Laboratory of Plant Hormones and Development Regulation of Chongqing, School of Life Sciences, Chongqing University, 401331 Chongqing, China.

${ }^{2}$ Center of Plant Functional Genomics, Institute of Advanced Interdisciplinary Studies, Chongqing University, 401331 Chongqing, China.

${ }^{3}$ College of Mathematics and statistics, Chongqing University, 401331 Chongqing, China.

\section{* Correspondences:}

Yudong Liu, yudongliu@cqu.edu.cn;

Zhiqiang Xian, xianzhiqiang@cqu.edu.cn;

Zhengguo Li, zhengguoli@ cqu.edu.cn. Tel and Fax, +86-23-65678902.

\section{Highlight}

The BRI1-EMS-SUPPRESSOR 1 (BES1) family protein SIBES1.8 promotes leaf simplification by inhibiting gibberellin deactivation and physically interacting with DELLA protein in tomato. 


\section{Abstract}

2 Leaf morphogenetic activity determines its shape diversity. However, our 3 knowledge to the regulatory mechanism in maintaining leaf morphogenetic capacity is

4 still limited. In tomato, gibberellin (GA) negatively regulates leaf complexity by 5 shortening the morphogenetic window. We here reported a tomato

6 BRI1-EMS-SUPPRESSOR 1 (BES1) transcription factor, SIBES1.8, that promoted

7 the simplification of leaf pattern in a similar manner as GA functions. Enhanced level

8 of SIBES1.8 dramatically decreased the sensibility of tomato to GA whereas increased

9 the sensibility to the GA biosynthesis inhibitor, PAC. In line with the phenotypic 10 observation, the endogenous bioactive GA contents were increased in OE-SIBES1.8

11 lines, which certainly promoted the degradation of the GA signaling negative 12 regulator, SIDELLA. Moreover, transcriptomic analysis uncovered a set of 13 overlapping genomic targets of SIBES1.8 and GA, and most of them were regulated 14 in the same way. Expression studies showed the repression of SIBES1.8 to the transcriptions of two GA deactivated genes, SlGA2ox2 and SlGA2ox6, and one GA receptor, SlGIDIb-1. Further experiments confirmed the direct regulation of SIBES1.8 to their promoters. On the other hand, SIDELLA physically interacted with SIBES1.8 and further inhibited its transcriptional regulation activity by abolishing SIBES1.8-DNA binding. Conclusively, by mediating GA deactivation and signaling, SIBES1.8 greatly influenced tomato leaf morphogenesis.

Keywords: BES1 transcription factor, leaf morphogenesis, gibberellin metabolism, gibberellin signaling, DELLA, tomato (Solanum lycopersicum). 


\section{Introduction}

Leaf is a vital plant organ not only because of its functions as a solar panel that utilizing light energy to converts carbon dioxide into carbohydrates, but also for the integration of internal and external signals in response to various environmental information (Du et al., 2018). Leaf primordia initiate from the peripheral zone (PZ) of shoot apical meristem (SAM), then the three axes of polar growth, that are the adaxial-abaxial, proximal-distal and medio-lateral axes, are gradually established (Byrne, 2012; Du et al., 2018). The margins of leaf primordia maintain a transient window of meristematic activity, allowing the production of leaflets, lobes and serrations, thus fine-tuning of this window enables the variability in leaf patterning (Alvarez et al., 2016; Israeli et al., 2021). According to the number of blade, leaves are classified as simple leaf that only has a single blade and compound leaf that owns several separate blades attached to a common rachis (Efroni et al., 2010). Tomato (Solanum lycopersicum) is a typical model plant that possesses compound leaves, which are composed of four kinds of leaflets: terminal leaflet (TL), primary lateral leaflet (PLL), secondary lateral leaflet (SLL) and intercalary leaflet (IL), and the lobes or serrations can be found in the margin of leaflet. There are usually one TL and three pairs of PLLs in tomato leaf branch. SLL develops from the petiolule of PLL, whereas IL develops later from the rachis between PLL, and their numbers are diverse and indeterminate (Goliber et al., 1999).

The initiation, differentiation and maturation of plant leaf are controlled by the combination of transcriptional regulators, microRNAs and phytohormones. The initiation of leaf primordia is required for the presence of auxin maxima, which are established by the auxin efflux carrier PINFORMED1 (PIN1) (Reinhardt et al., 2000; Reinhardt et al., 2003). Late study reported that auxin induced the expression of a tomato ortholog of Arabidopsis DORNRONSCHEN (DRN) gene, LEAFLESS (LFS), which is necessary for leaf initiation, to specify primordium cells in the PZ (Capua and Eshed, 2017). Besides, many transcription factors are involved in the regulation of leaf initiation, including class I KNOTTED1-like homeobox proteins (KNOXI) and 
the ASYMMETRIC LEAVES1 (AS1)/ROUGH SHEATH2 (RS2)/PHANTASTICA (ARP) MYB-domain transcription factors (Kerstetter et al., 1997; Byrne et al., 2000; Lodha et al., 2013). During leaf patterning, a set of regulating factors including MONOPTEROS (MP, also named ARF5), GOBLET (GOB), LYRATE (LYR) and REDUCED COMPLEXITY (RCO) coordinately modulate the formation and shaping of leaflet in parallel different aspects (Vlad et al., 2014; Israeli et al., 2021). Leaf patterning is also greatly regulated by several miRNA-target modules. For instance, miR165/166 contributes to the establishment of leaf adaxial-abaxial polarity by targeting HD-ZIP $\square$ mRNAs (Tatematsu et al., 2015; Merelo et al., 2016), miR319 and miR396 antagonistically regulate leaf marginal cell proliferation by targeting the class $\square$ TEOSINTE BRANCHED1/CYCLOIDEA/PROLIFERATING CELL FACTOR (TCP) and GROWTH-REGULATING FACTORs (GRFs) respectively (Ori et al., 2007; Rodriguez et al., 2010; Tsukaya, 2018).

Leaf morphogenesis is also regulated by several phytohormones (Shwartz et al., 2016). Among them, Gibberellin (GA) is shown to reduce the complexity of tomato leaf. Exogenous GA application promotes the maturation of tomato leaf and inhibits the formation of leaflets. Similarly, leaves of the procera (pro) mutant, which sustained mutation in the single tomato DELLA gene and showed constitutive GA response, bear fewer leaflets with smooth margins (Jasinski et al., 2008; Lombardi-Crestana et al., 2012; Shwartz et al., 2016). This leaf simplification caused by increased GA levels or responses indicates the role of GA in shortening the morphogenetic window during leaf formation. Some leaf morphogenesis regulators are shown to function in mediating GA dynamics. In tomato, the CIN-TCP transcription factor LANCEOLATE (LA) accelerates leaf maturation and simplifies leaf shape partially by positively regulating GA levels (Yanai et al., 2011). By contrast, KNOXI protein antagonizes GA activity by repressing the expression of GA biosynthesis gene SlGA20oxl in regulating leaf morphogenesis (Jasinski et al., 2008). In addition to GAs, Brassinosteroids (BRs) are another kind of plant growth-promoting hormone with functions in leaf development by promoting cell 
82

83

84

85

86

87

88

89

90

91

92

93

94

95

96

97

98

99

100

101

102

103

104

105

106

107

108

109

110

elongation and differentiation (Mitchell et al., 1970; Grove et al., 1979). BR biosynthetic and signaling mutants indeed show abnormal leaf patterning (Koka et al., 2000; Zhiponova et al., 2013). The crosstalk between GAs and BRs was well explored in the last decade. In Arabidopsis, the negative regulators of GA signaling, DELLA proteins, physically interacted with the dephosphorylated BRASSINAZOLE RESISTANT1 (BZR1), the key positive regulator of BR signaling (Bai et al., 2012; Gallego-Bartolomé et al., 2012; Li et al., 2012). Moreover, the transcriptional regulation activity of BZR1 was inhibited by the interaction with DELLA proteins (Bai et al., 2012; Gallego-Bartolomé et al., 2012; Li et al., 2012). On the other hand, genetic and biochemical evidences suggested that BZR1 and BRI1-EMS-SUPPRESSOR 1 (BES1) affected GA levels by directly regulating the expression of GA biosynthetic genes GA20ox and GA3ox in Arabidopsis and rice (Tong et al., 2014; Unterholzner et al., 2015). This integration of GA and BR both on signaling level and hormone level allowed the elaborated regulation of plant growth and leaf development.

Here, we reported a tomato BES1 family member, SIBES1.8, that can influence leaf morphogenesis by mediating GA deactivation and signaling. Overexpression of SIBES1.8 in tomato significantly reduced the leaf complexity, which mimicked the functions of GA in regulating leaf development. Indeed, the endogenous bioactive GA contents were increased in OE-SIBES1.8 leaves because of the transcriptional repression of SlGA2ox2 and SlGA2ox6 by SIBES1.8, which further resulted in the degradation of SIDELLA, hence the GA signaling was enhanced in OE-SIBES1.8 lines. On the other hand, the transcriptional regulation ability of SIBES1.8 was inhibited by SIDELLA interaction, and such inhibition was released by the SIBES1.8-promoted degradation of SIDELLA, which further enhanced GA signaling in OE-SlBES1.8 lines. Together, our results provide new insight into the crosstalk between BES1 transcription factors and GA levels or responses in regulating leaf morphogenesis. 


\section{Materials and Methods}

\section{Plant material growth conditions and generation of SIBES1.8 over-expressed lines}

The coding sequence of SIBES1.8 was cloned into K303 binary vector under the promotion of CaMV 35S promoter (Ren et al., 2011; Liu et al., 2020). The recombined expression vector was transformed into Agrobacterium tumefaciens strain GV3101 followed by cotyledon transformation according to V J Chetty et al. (2013) in tomato (Solanum lycopersicum) cv. Micro-Tom and Ailsa Craig background to generate the OE-SIBES1.8 lines. Positive transgenic lines were screened by kanamycin and PCR detection, and the relative mRNA level of SIBES1.8 in positive lines was detected by quantitative real-time polymerase chain reaction (qRT-PCR) in T2 generation. All tomato plants were cultivated in greenhouse with 16/8 h light/dark cycle, $25 / 20 \square$ day/night temperature and $60 \%$ relative humidity.

\section{Observation of leaf pattern by scanning electron microscopy (SEM)}

The extra leaves of WT and OE-SIBES1.8 plants at 15 DPG were hand-dissected to expose the leaf primordium. The shoots of seedlings were subsequently immersed into FAGE solution (50\% absolute ethanol, 10\% formaldehyde, 5\% acetic acid and $0.72 \%$ glutaraldehyde) immediately for one day followed by dehydrating in an ethanol series from $30 \%$ to $100 \%$ over five days. The prepared samples were critically point dried and coated with gold. Finally, the images were taken by a FEI Inspect S50 scanning electron microscope.

\section{Gibberellin A3 (GA3) and paclobutrazol (PAC) treatment}

$\mathrm{GA}_{3}$ and PAC (Sigma, USA) were dissolved in absolute methanol. For $\mathrm{GA}_{3}$ and PAC treatment, tomato seeds were germinated on MS/2 medium containing $\mathrm{GA}_{3}(10$ $\mu \mathrm{M}$ and $50 \mu \mathrm{M})$ or PAC $(5 \mu \mathrm{M})$ respectively after sterilization. The control seeds were germinated on MS/2 medium containing the same solution without $\mathrm{GA}_{3}$ and PAC. After 10-days normal growth, the seedlings were transplanted to the soil and sprayed with $100 \mu \mathrm{M} \mathrm{GA}_{3}$ or $50 \mu \mathrm{M}$ PAC solution twice a week. Plant samples were observed 
or collected at the corresponding times as described in the context.

\section{Measurement of endogenous GAs contents}

143 For measuring endogenous GAs contents, leaves of WT and OE-SlBES1.8 plants at 144 5-week-old were harvested and frozen into liquid nitrogen immediately. The 145 measurement was conducted by Wuhan Metware Biotechnology Co., Ltd. (Wuhan, 146 China) based on the AB Sciex QTRAP 6500 LC-MS/MS platform. Briefly, leaf 147 samples were ground using a mixer mill (MM 400, Retsch, Germany) to powder. GAs 148 were extracted with $70 \%(\mathrm{v} / \mathrm{v})$ acetonitrile from $500 \mathrm{mg}$ powder. After adding the 149 internal standards, $100 \mu \mathrm{L}$ trimethylamine and $100 \mu \mathrm{L}$ BPTAB, the resulting solution was vortexed, incubated at $90 \square$ for 1 hour, and evaporated to dryness under nitrogen gas stream followed by redissolving in $1 \mathrm{~mL} \mathrm{70 \%} \mathrm{(v/v)} \mathrm{acetonitrile.} \mathrm{The} \mathrm{extracts} \mathrm{were}$ filtered through $0.22 \mu \mathrm{m}$ filter and then analyzed using an UPLC-ESI-MS/MS system (UPLC, ExionLCTM AD, https://sciex.com.cn/; MS, Applied Biosystems 6500 Triple Quadrupole, https://sciex.com.cn/). Finally, the output data was analyzed by Analyst 1.6.3 software.

\section{RNA extraction, cDNA synthesis and qRT-PCR analysis}

Total RNA was extracted by using the RNeasy Plant Mini Kit (Qiagen, Germany) and treated with RNase-free DNase (Qiagen, Germany). The integrity and concentration of total RNA were detected by agarose gel electrophoresis and NanoDrop 1000 (Thermo, USA) respectively. After that, the PrimeScript ${ }^{\mathrm{TM}}$ RT reagent Kit with gDNA Eraser (TAKARA, Japan) was used to synthesize the first-strand cDNA with $1 \mu \mathrm{g}$ total RNA as the template. The cDNA products were diluted to 5-fold with deionized water before use. qRT-PCR was performed by TB Green ${ }^{\circledR}$ Premix Ex Taq ${ }^{\text {TM }} \square$ (Tli RNaseH Plus) (TAKARA, Japan) on the CFX96 Touch $^{\mathrm{TM}}$ Real-Time PCR Detection System (BIO-RAD, USA) according to the manufacturer's instructions. The relative fold change was calculated by the $2^{-\Delta \Delta \mathrm{Ct}}$ method. SIUBI (Solyc01g056940) was used as the internal reference gene and all 
primers used in qRT-PCR detection were listed in Supplementary Table S1.

\section{RNA sequencing and data processing}

172 The fourth leaf primordium (P4) of WT and OE-SlBES1.8 seedlings at 16 DPG 173 (that is L6 presented in Fig. 2) treated with or without $50 \mu \mathrm{M} \mathrm{GA}_{3}$ was microdissected 174 with a surgical blade and frozen into liquid nitrogen immediately, which was regarded 175 as the leaf samples used in RNA sequencing. At least 30 leaf primordia were collected 176 for each sample, and three independent samples were sequenced for each group. The 177 total RNA was extracted by using the PicoPure ${ }^{\mathrm{TM}}$ RNA Extraction kit (ARCTURUS, 178 USA) according to the user guide. The concentration of total RNA was measured by 179 NanoDrop 1000 (Thermo, USA). The integrity of total RNA was detected by agarose gel electrophoresis and 2100 Bioanalyser (Agilent, USA) and only those RNA samples showed clear bands and high RNA Integrity Number were conducted sequencing library. The RNA-seq was carried out in Majorbio (Shanghai Majorbio Bio-pharm Technology, Ltd, China) with the Illumina HiSeq xten/Novaseq 6000 sequencer. The raw data were trimmed and qualified by SeqPrep and Sickle with default parameters. The generated clean reads were subsequently mapped to the tomato genome in the Solanaceae Genomics Network (http://solgenomics.net/) by using HISAT2 software. Finally, DESeq2 was used to analyze the differentially expressed genes (DEGs) with fold change (FC) $>2$ and adjust $P$-value $(Q$-value) $<$ 0.05 .

\section{Electrophoretic mobility shift assay (EMSA)}

192 Full length of SIBES1.8 and SIDELLA coding sequences were cloned into pGEX-4T-1 plasmid. The recombined plasmids were expressed in Escherichia coli strain Rosetta2 BL21 cells (Transgen, China) to produce the GST fused proteins. The fused proteins were purified with the GST-tagged protein purification kit (Clontech, USA) according to the manufacturer's instructions. The designed probes of SlGA2ox2, 
198

Shift ${ }^{\circledR}$ Chemiluminescent EMSA Kit (Thermo, USA). The mutated biotin probes that had an AAAAAA fragment instead of G-box were used to confirm the specific binding and the unlabeled probes were used as the competitor. For assessing the effect of SIDELLA to the DNA binding ability of SIBES1.8, SIBES1.8 and SIDELLA proteins were mixed before EMSA reaction. All native and mutated probes were listed in Supplementary Table S1.

\section{Yeast one-hybrid (Y1H) assays}

The promoter fragments of SlGA2ox2, SlGA2ox6 and SlGID1b-1 were constructed into pAbAi plasmid and transformed into Y1HGold yeast strain under the guidance of Yeastmaker ${ }^{\mathrm{TM}}$ Yeast Transformation System 2 (TAKARA, Japan). The recombinant yeast strain was confirmed by PCR conducted with Matchmaker ${ }^{\mathrm{TM}}$ Insert Check PCR Mix (TAKARA, Japan). The full length of SIBES1.8 coding sequence was cloned into pGADT7 plasmid and subsequently transformed into the recombinant yeast strain. The empty pGADT7 plasmid was used as negative control. The transformants were cultivated on SD/-Leu or SD/-Leu/AbA (Aureobasidin A, Clontech, USA) medium for three days. DNA-protein interactions between S1BES1.8 and promoter fragments were determined by the growth of colony.

\section{Dual-luciferase assays}

To explore the direct transcriptional regulation of SlBES1.8 to SlGA2ox2, SlGA2ox6 and SIGID1b-1, full length of SIBES1.8 coding sequence was cloned into pGreen $\square 62-S K$ to generate the effector, and promoter fragments of SlGA2ox2, SlGA2ox6 and SlGIDIb-1 were constructed into pGreen II 0800-LUC respectively to generate the reporters (Hellens et al., 2005). After transiently co-expressed the effector and reporter in tobacco leaf for one-day darkness and another two-days normal growth, the expression of $L U C$ and $R E N$ were detected by the Dual-Luciferase Reporter Assay System (Promega, USA). At least six biological replicates were measured, and the detection was performed independently for two times with similar 
results.

To explore the effect of SIDELLA on the transcriptional repression activity of SIBES1.8, full length of SIBES1.8 and SIDELLA coding sequences were cloned into pEAQ-GAL4BD vector respectively to generate the effectors. In reporter, the expression of firefly luciferase (LUC) was under the control of GAL4-binding element (5xGAL4), which was fused with the minimal TATA region of CaMV35S, and the renilla luciferase (REN) promoted by CaMV35S was considered as the internal control. The SIBES1.8 and SIDELLA effectors were separately or together co-expressed with the reporter in tobacco leaves. Finally, the $L U C$ and $R E N$ expression were detected by the Dual-Luciferase Reporter Assay System (Promega, USA). At least six biological replicates were measured, and the detection was performed independently for two times with similar results.

\section{Immunoblot assay}

For immunoblot analyses, 16-days-old tomato seedlings were sprayed with $100 \mu \mathrm{M}$ $\mathrm{GA}_{3}$ or $50 \mu \mathrm{M}$ PAC solution for 3 hours, and then frozen into liquid nitrogen immediately. Protein extracts were performed with the Plant Total Protein Extraction Kit (Sigma, USA). Protein concentrations were measured using the BCA method. Equal amounts of protein from each sample were boiled in $4 \times$ Laemmli protein sample buffer (Bio-rad, USA) for $10 \mathrm{~min}$ followed by separating with $10 \%$ SDS-PAGE. After a wet transfer to PVDF membranes, the anti-GAI rabbit antibody and goat anti-rabbit IgG antibody (Agrisera, Sweden) were used to perform immunoblot.

\section{Yeast two-hybrid (Y2H) assays}

Full length of SIBES1.8 and SIDELLA coding sequences were constructed into pGBKT7 and pGADT7 as bait and prey respectively. The recombined plasmids were co-transformed into Y2H Gold yeast cells. Culture mediums SD/-Leu/-Trp and SD/-Ade/-His/-Leu/-Trp (Clontech, USA) with or without X- $\alpha$-gal were used to select 
256

257

258

259

260

261

262

263

264

265

266

267

268

269

270

271

272

273

274

275

276

277

278

279

280

281

282

283

284

the positive transformants.

\section{Bimolecular fluorescence complementation (BiFC) assays}

Full length of SIBES1.8 and SIDELLA coding sequences were cloned into pXY104-YFP ${ }^{\mathrm{C}}$ and pXY106-YFP ${ }^{\mathrm{N}}$ (Yu et al., 2008) respectively. The BiFC assays were performed as described previously (Luo et al., 2014). In short, the recombined plasmids were transformed into Agrobacterium tumefaciens strain GV3101 and co-injected into and expressed in tobacco epidermic cells. The recombined plasmids co-injected with corresponding empty $\mathrm{YFP}^{\mathrm{N}}$ or $\mathrm{YFP}^{\mathrm{C}}$ were used as negative control. After incubated in darkness for one day and in normal light condition for another two days, the fluorescence images were captured by the laser scanning confocal microscope (Leica TCS SP8, Germany).

\section{Firefly luciferase complementation imaging (LCI) assays}

Full length or truncated versions of SIBES1.8 and SIDELLA were fused with nLUC or cLUC respectively. The LCI assays were conducted following the description by Chen et al. (2008). Briefly, the recombined plasmids were transformed into Agrobacterium tumefaciens strain GV3101. The fused S1BES1.8-nLUC transformants were transiently co-expressed with cLUC-SIDELLA transformants in tobacco leaves. After incubated in darkness for one day and in normal light condition for another two days, the tobacco leaves were sprayed with one millimolar luciferin (Promega, USA) on the blade back and kept in dark at least for six minutes. Finally, the LCI images were captured by a low-light cooled CCD imaging apparatus (Alliance, UK). At least four leaves were observed for each combination.

\section{Accession numbers}

Arabidopsis genes are obtained from The Arabidopsis Information Resource (TAIR, https://www.arabidopsis.org/) under the following identities: GAI, AT1G14920; RGA, AT2G01570; $R G L 1$, AT1G66350; RGL2, AT3G03450; RGL3, AT5G17490. Tomato 
285

286

287

288

289

290

291

292

293

294

295

296

297

298

299

300

301

302

303

304

305

306

307

308

309

310

311

312

313

genes are collected from Solanaceae Genomics Database (http://solgenomics.net/) under the following accession numbers: SlBES1.8, Solyc10g076390; SlGA2ox2, Solyc07g056670; SlGA2ox6, Solyc01g058030; SlGID1b-1, Solyc09g074270; SIDELLA, Solyc11g011260; SlGA2ox1, Solyc05g053340; SlGA2ox3, Solyc01g079200; SlGA2ox4, Solyc07g061720; SlGA2ox5, Solyc07g061730; SlGA2ox7, Solyc02g070430; SlGA2ox8, Solyc08g016660; SlGA2ox9, Solyc10g007570; SlGIDlac, Solyc01g098390; SlGID1b-2, Solyc06g008870; SlSLY1, Solyc04g078390; SISNE, Solyc07g047680; SIUBI, Solyc01g056940. The raw RNA-sequencing data are publicly available in the Genome Sequence Archive in National Genomics Data Center, Beijing Institute of Genomics, Chinese Academy of Sciences with the accession number of CRA005324.

\section{Results}

SlBES1.8 mimics the effect of exogenous GA application in simplifying leaf complexity

Previously, we reported that SIBES1.8 exhibited distinct gene structure compared to other BES1 members in tomato and acted as a transcriptional repressor with floral tissues specific expression pattern (Su et al., 2021). To explore the functions of SIBES1.8 in plant development, we generated over-expressed (OE) lines driving by CaMV35S promoter in micro-tom background. Three OE-SlBES1.8 lines (L4, L5 and L8) with dramatically increased SIBES1.8 expressions were selected to analyze the phenotypic changes (Supplementary Fig. S1A). Remarkably, OE-SlBES1.8 plants exhibited simpler leaf shape with smooth margin compared with wild type (WT) plants, and this phenotypic change occurred in early leaf primordium development (Fig. 1A-C). In WT leaf primordium, serrations were emerged from the margin of leaflet, while there was no such structure in OE-SIBES1.8 leaf primordium (Fig. 1A, B). Consistent with this, the serration numbers of mature terminal leaflets and primary lateral leaflets were significantly decreased in OE-SIBES1.8 leaves (Fig. 1C). Given that micro-tom is a dwarf cultivar of tomato and its leaves are simplified by the 
314 mutation in the SELF-PRUNING $(S P)$ and DWARF $(D)$ genes, which are related with

315 shoot apical meristem development and BR biosynthesis respectively (Martí et al., 316 2006), we also generated OE-SIBES1.8 lines in Ailsa Craig background

317 (Supplementary Fig. S1B), in which the leaf pattern remained complicated. Consistent

318 with the observation in micro-tom background, OE-SlBES1.8 leaves also showed 319 simpler shape and smooth margin (Fig. 1D, Supplementary Fig. S2). The leaflets can 320 be briefly classified into three groups: terminal leaflet and primary lateral leaflets (1st), 321 secondary lateral leaflets (2nd) and intercalary leaflets (3rd) (Fig. 1E). The numbers 322 of all of these three kinds of leaflets were decreased in OE-SIBES1.8 leaves (Fig. 1F). 323 On the contrary, we also generated slbes 1.8 mutants by CRISPR/Cas9 technology. 324 Two homozygous lines with distinct mutations were selected to analyze the leaf 325 pattern (Supplementary Fig. S3A), while no obvious phenotypic changes were 326 observed (Supplementary Fig. S3B), and the leaflet serration numbers of the $5^{\text {th }}$ leaf 327 were not significantly influenced in slbes 1.8 mutants (Supplementary Fig. S3C), 328 indicating that knockout of SIBES1.8 didn't affect the normal growth of tomato leaf 329 development.

330 The decreased leaf complexity in OE-SLBES1.8 plant is reminiscent of the functions 331 of GA in leaf development that increased GA levels or responses could predominantly 332 simplify leaf shape (Jasinski et al., 2008; Yanai et al., 2011; Lombardi-Crestana et al., 333 2012; Livne et al., 2015; Tomlinson et al., 2019). To compare the similarity of leaf 334 phenotypic changes caused by OE-SIBES1.8 and GA application, we treated wild type 335 seedlings with $\mathrm{GA}_{3}$ and observed the leaf shape. The results showed that WT leaves 336 possessed several serrations in the margin (Fig. 2A). This serration structure can be 337 firstly observed in L6 (the $4^{\text {th }}$ leaf primordium, P4), while no such structure can be 338 found after applying exogenous $\mathrm{GA}_{3}$ (Fig. 2A, B). In OE-SlBES1.8 seedlings, there is 339 also no serration structure taken shape (Fig. 2C). Phenotypic observation in mature 340 leaves further confirmed the consistent roles of $\mathrm{GA}_{3}$ and SIBES1.8 in leaf 341 development (Supplementary Fig. S4). These results indicate that OE-SlBES1.8 342 altered the leaf morphogenesis in a manner that similar to GA application. 
SIBES1.8 decreases the sensibility of tomato to exogenous $\mathrm{GA}_{3}$ while increases the sensibility to PAC

346 The similarity in shaping leaf promotes the possibility that SIBES1.8 may have 347 direct or indirect correlation with GA. We first analyzed the potential cis-elements in 348 the promoter of SIBES1.8 and found that there was a GA responsive TATC-box (Fig. 349 3A). Moreover, qRT-PCR detection verified the induction of $\mathrm{GA}_{3}$ to the transcription 350 of SlBES1.8 (Fig. 3B). The primary root of OE-SlBES1.8 seedlings was shorter than 351 WT and was not influenced by $\mathrm{GA}_{3}$ treatment (Fig. 3C, D). In addition, the most 352 obvious phenotypic change of tomato seedling after $\mathrm{GA}_{3}$ treatment is the elongation 353 of first shoot (Fig. 3C). Surprisingly, the GA-induced first shoot elongation was 354 suppressed in OE-SIBES1.8 lines (Fig. 3C, E). These results suggested that SlBES1.8 355 decreased the sensibility of tomato seedling to exogenous $\mathrm{GA}_{3}$. On the other hand, paclobutrazol (PAC, an inhibitor of GA biosynthesis) treatment could suppress plant growth and produce smaller leaves (Fig. 3F). While the growth suppression of PAC to OE-SlBES1.8 plants was much stronger than WT plants (Fig. 3F). These results indicated that SIBES1.8 increased the sensibility of tomato to PAC. Noticeably, PAC treatment partly recovered the serration in OE-SIBES1.8 leaf margin (Fig. 3F), which further tied SIBES1.8 functions with GA level or response in leaf development.

\section{SlBES1.8 increases endogenous bioactive GA contents in tomato leaf}

To assess the effect of OE-SlBES1.8 to GA levels, we detected the endogenous GA contents of WT and OE-SIBES1.8 leaves. According to the GA biosynthesis pathway, we measured the endogenous contents of 16 kinds of GAs in mature leaves, including $\mathrm{GA}_{15}, \mathrm{GA}_{9}, \mathrm{GA}_{51}, \mathrm{GA}_{4}, \mathrm{GA}_{34}, \mathrm{GA}_{20}, \mathrm{GA}_{1}, \mathrm{GA}_{8}, \mathrm{GA}_{5}, \mathrm{GA}_{3}, \mathrm{GA}_{7}, \mathrm{GA}_{24}, \mathrm{GA}_{53}, \mathrm{GA}_{19}$,

$368 \mathrm{GA}_{29}$ and $\mathrm{GA}_{6}$ (Fig. 4A, B, Supplementary Table S2). Among them, the contents of $\mathrm{GA}_{24}, \mathrm{GA}_{53}, \mathrm{GA}_{19}, \mathrm{GA}_{29}$ and $\mathrm{GA}_{6}$ were too low to detect. In general, total GAs contents were not significantly changed in OE-SIBES1.8 leaves (Fig. 4C), while the 
372 decreased respectively (Fig. 4B, D). Furthermore, the other two bioactive GAs, GA

373 and $\mathrm{GA}_{4}$ were unchanged and increased respectively in OE-SlBES1.8 leaves (Fig. 4E).

374 These results suggested that the increased bioactive GA levels may account for the 375 decreased complexity of OE-SIBES1.8 leaf.

\section{SIBES1.8 and GA regulate overlapping genomic targets}

To view insight of the genome-wide transcriptomic profiling of OE-SlBES1.8 plants, we performed RNA-sequencing for WT and OE-SIBES1.8 leaf primordium at P4 stage. With the criterions of fold change (FC) $>2$ and adjust $P$-value $(Q)<0.05$, a total of 259 and 297 differentially expressed genes (DEGs) were influenced by $\mathrm{GA}_{3}$ application and OE-SIBES1.8 respectively (Fig. 5A, Supplementary Table S3, 4). Among them, 42 DEGs were regulated by both $\mathrm{GA}_{3}$ and SIBES1.8, which promoted the close relationship between $\mathrm{GA}_{3}$ and SIBES1.8 (Fig. 5B, Supplementary Table S5). If SIBES1.8 mimics the functions of GA in leaf development, SIBES1.8 should affect the expression of genes that influenced by GA in a similar manner. Indeed, by analyzing the expression profiles of these 42 co-DEGs, we found that 36 DEGs $(85.7 \%)$ were affected in the same way by $\mathrm{GA}_{3}$ and OE-SIBES1.8 (Fig. 5C, group $\square$ ), whereas only six DEGs were influenced in the opposite way (Fig. 5C, group $\square$ ). Notably, when compared the expression of these co-DEGs in $\mathrm{GA}_{3}$ treated OE-SlBES1.8 leaves with mocked WT leaves (OE GA 3 vs WT Mock), the expression changes displayed a superimposed effect of OE-SIBES1.8 and $\mathrm{GA}_{3}$ treatment, which suggested that on the basis of exogenous $\mathrm{GA}_{3}$ application, OE-SlBES1.8 further strengthened GA signaling (Fig. 5C). Meanwhile, by comparing the expression of these 42 DEGs in WT and OE-SIBES1.8 leaves after $\mathrm{GA}_{3}$ treatment, we found that 28 DEGs (66.7\%, group $\square$ ) exhibited weaker expression changes in OE-SIBES1.8 leaves than that in WT (Fig. 5D), suggesting that OE-SlBES1.8 repressed their responses to

$\mathrm{GA}_{3}$ application, which further supported our previous conclusion that SlBES1.8 399 decreased the sensibility of tomato to exogenous GA 3 . Collectively, OE-SIBES1.8 and 
SlBES1.8 binds to and represses the activities of SIGA2ox2, SIGA2ox6 and

\section{SIGID1b-1 promoters}

404

To elucidate the regulatory mechanism of SIBES1.8 in regulating leaf development by mediating GA levels or responses, we carefully analyzed all DEGs affected by OE-SlBES1.8 and $\mathrm{GA}_{3}$ application, and found that merely none of them was reported to involve in GA biosynthesis or signaling (Supplementary Table S3, 4). This may be caused by the very early developmental status of RNA-seq samples (the $4^{\text {th }}$ leaf primordium of seedlings at 16DPG) that few of genes exhibited differential expression in this stage. Hence, we selected to explore the potential targets of SIBES1.8 by using the older leaves (that is L3 presented in Fig. 2) as the materials.

The increased bioactive GA contents could be caused by the up-regulation of GA biosynthetic genes or the down-regulation of GA deactivated genes. Given that SIBES1.8 acts as a transcriptional repressor (Su et al., 2021), we mainly considered the repression of SIBES1.8 to the expression and responsiveness of GA 2-oxidase $(G A 2 o x)$ genes, which are the best-characterized GA deactivated genes. The relative expressions of nine tomato GA2ox genes in WT and OE-SlBES1.8 leaves with or without $\mathrm{GA}_{3}$ treatment were explored by qRT-PCR. Overall, SlGA2oxs transcriptions were greatly induced by $\mathrm{GA}_{3}$ in WT leaves as expected (Fig. 6A, B, Supplementary Fig. S5A). Among them, the expression levels of SlGA2ox2 and SlGA2ox6 were significantly reduced in OE-SIBES1.8 leaves, and their responses to $\mathrm{GA}_{3}$ application were also suppressed by OE-SIBES1.8 (Fig. 6A, B), suggesting that SIBES1.8 may increase the bioactive GA contents through repressing the expressions of SlGA2ox2 and SlGA2ox6. Meanwhile, we also detected the relative expression of genes involved in GA signaling (Fig. 6C, Supplementary Fig. S5B). Among them, SlGID1b-1 showed reduced expression in OE-SIBES1.8 leaves (Fig. 6C), indicating a potential direct regulation by SIBES1.8.

428 To confirm the direct regulation of SIBES1.8 to SlGA2ox2, SlGA2ox6 and 429 SlGID1b-1, we conducted electrophoretic mobility shift assay (EMSA), yeast 
one-hybrid assay $(\mathrm{Y} 1 \mathrm{H})$ and dual-luciferase assay according to the existence of G-box (CACGTG), a well-identified binding motif of BES1 family (He et al., 2005; Yin et $a l .$, 2005), in the promoters of SlGA2ox2, SlGA2ox6 and SlGIDIb-1 (Fig. 6D). EMSA results showed the specific binding of SIBES1.8 to the selected promoter fragments (Fig. 6E). Y1H results further confirmed the binding of S1BES1.8 to SlGA2ox2, SlGA2ox6 and SlGIDIb-1 promoters (Fig. 6F). Moreover, we used dual-luciferase system to detect the effects of SIBES1.8 to the promoter activities in tobacco leaves and found that SIBES1.8 significantly repressed the activities of SlGA2ox2, SlGA2ox6 and SlGIDIb-1 promoters (Fig. $6 \mathrm{G}, \mathrm{H}$ ). The transcriptional repression of SIBES1.8 to SlGA2ox2 and SlGA2ox6 may be the reason of increased bioactive GA contents in OE-SIBES1.8 leaves, which should promote the degradation of SIDELLA protein. Indeed, by performing immunoblot, we confirmed the decreased level of SIDELLA protein in OE-SlBES1.8 lines (Fig. 6I). Collectively, these results indicated that SIBES1.8 increased the endogenous bioactive GA levels by repressing the expression of SlGA2ox2 and SlGA2ox6, which further promoted the degradation of SIDELLA protein, resulting in a similar effect with increased GA levels or responses in tomato leaf development.

\section{SIDELLA physically interacts with SlBES1.8 and inhibits its transcriptional regulation ability by abolishing SIBES1.8-DNA binding}

DELLA protein lacks a DNA-binding domain, which promotes the necessary of intermediate proteins for its functions (Yoshida et al., 2014; Marín-de la Rosa et al., 2015). In Arabidopsis, DELLAs were proved to physically interact with BES1/BZR1, which further inhibited the DNA binding ability of BZR1 (Bai et al., 2012; Gallego-Bartolomé et al., 2012; Li et al., 2012). The characteristic domains of DELLA proteins were conserved in tomato and Arabidopsis (Supplementary Fig. S6), which raised the possibility that tomato DELLA protein may interact with SIBES1 transcription factors in a similar manner, including SIBES1.8. To prove this assumption, we investigated the protein-protein interaction between SIDELLA and 
SIBES1.8 by yeast two-hybrid assay (Y2H), bimolecular fluorescence complementation (BiFC) assay and firefly luciferase complementation imaging (LCI) assay. The results showed that SIDELLA can truly interact with SIBES1.8 (Fig. 7A-C). Furthermore, we explored the interacted domains of SIDELLA and SIBES1.8 by generating a series of deletion constructs used in LCI assays according to the amino acid sequence analysis (Supplementary Fig. S7A). The results showed that the deletion of PEST domain of SIBES1.8 was sufficient to prevent the interaction with SIDELLA (Supplementary Fig. S7B), and deletion of DELLA motif of SIDELLA abolished the interaction with SIBES1.8 (Supplementary Fig. S7C), indicating that the PEST domain of SIBES1.8 and the DELLA motif of SIDELLA were critical for their protein-protein interaction.

We next analyzed the effect of SIDELLA interaction to the transcriptional regulation activity of SIBES1.8 by GAL4-responsive reporter system in tobacco leaves. Full length of SIDELLA and SIBES1.8 coding sequences were fused with GAL4BD respectively to generate the effectors, which were subsequently co-expressed with reporter in tobacco leaves (Fig. 7D). As the transcriptional activation control, VP16 greatly induced the expression of $L U C$, indicating the effectiveness of this system. In line with our previous detection ( $\mathrm{Su}$ et al., 2021), SIBES1.8 significantly repressed $L U C$ expression, suggesting the transcriptional repression activity. On the contrary, SIDELLA increased LUC expression and exhibited transactivation activity, which was consistent with the rice DELLA protein, SLR1 (Hirano et al., 2012). Dramatically, SIDELLA antagonized the transcriptional regulation activity of SIBES1.8 when they co-expressed together (Fig. 7E). Besides, using EMSA, we confirmed that the bindings of SIBES1.8 to the promoter fragments of SlGA2ox2, SlGA2ox6 and SlGID1b-1 were suppressed by the presence of SIDELLA (Fig. 7F). Noticeably, SIDELLA was unable to bind these fragments in the absence of SIBES1.8, as expected. Taken together, these results indicated that through protein-protein interaction, SIDELLA can inhibit the transcriptional regulation activity of SIBES1.8 by repressing its DNA binding ability. 


\section{Discussion}

Leaf morphological diversity is achieved by fine-tuning the morphogenetic window during development that prolonged window resulted in complex leaf patterning whereas shortened window led to simpler leaf shape (Kierzkowski et al., 2019).

During this elaborated developmental process, several regulators acted coordinately to affect different aspects of leaf shape, ensuring the establishment of a suitable pattern (Du et al., 2018; Israeli et al., 2021). Hormones play critical roles in various aspects of plant development including leaf formation (Shwartz et al., 2016). Among them, GA is known to reduce the complexity of leaf pattern in tomato. Tomato plants treated with exogenous GA or mutated in DELLA locus exhibited simplified leaf that with less leaflet production and smooth margin (Jasinski et al., 2008; Lombardi-Crestana et al., 2012; Livne et al., 2015; Shwartz et al., 2016; Tomlinson et al., 2019). By investigating the early leaf development of tomato DELLA mutant pro, the simplification of pro leaf was caused by the combination of accelerated early developmental growth and delayed leaflet initiation (Jasinski et al., 2008), indicating the effects of increased GA signaling in shortening leaf morphogenetic phase. Since the clarification of GA functions in leaf development, several regulators that can greatly influence leaf morphogenesis were reported to fulfill their roles by affecting GA homeostasis, such as LA and KNOXI (Jasinski et al., 2008; Yanai et al., 2011). In this research, we also identified a tomato leaf development regulator, SIBES1.8, that promoted leaf simplification by mediating GA homeostasis and signaling. We offered multiple lines of evidence to support this finding. First, OE-SIBES1.8 lines in

511 micro-tom and Ailsa Craig background presented less leaflet with smooth margin,

512 which was highly resemble to GA-treated wild type leaves. Second, endogenous

513 bioactive GA levels were increased in OE-SIBES1.8 leaves, which subsequently led to 514 the degradation of SIDELLA as expected. Third, OE-SIBES1.8 and GA affected a set 515 of overlapping targets in a similar manner. Fourth, S1BES1.8 directly regulated the 
517 interacted with SIDELLA and were inhibited by such interaction in DNA binding

518 ability. In summary, we proposed a working model to depict the molecular mechanism

519 of SIBES1.8 in regulating tomato leaf morphogenesis by mediating GA deactivation

520 and signaling (Fig. 8). Although we determined the correlation of SIBES1.8 with GA,

521 the downstream targets of GA signaling that directly contribute to leaf development

522 remain largely unknown and need to be investigated. Also, new modifiers of leaf

523 pattern that affect variable aspects of leaf shape such as lobe/serration, leaflet number

524 and size, are worth to be discovered.

525 To identify the functions of SIBES1.8, we also generated CRISPR/Cas9 mutants for 526 it and expected the corresponding phenotypic changes to OE-SIBES1.8 plants in leaf 527 development. We selected two homozygous lines with distinct mutations to observe 528 the leaf phenotype and failed to obtain obvious developmental differences 529 (Supplementary Fig. S3). Two reasons may explain this. First, the constitutive 530 expression level of SIBES1.8 is very low, with an average of 0.86 TPM transcription 531 in wild type leaves, as a contrast, its transcription was increased to 1555.67 TPM in 532 OE-SlBES1.8 leaves (Supplementary Table S4). The low content of SIBES1.8 533 contributed to a weak repression to SlGA2ox2 and SlGA2ox6 transcription that its 534 knockout was not enough to affect endogenous GA levels. Second, decreasing GA 535 levels or responses contributed a relative minor effect on leaf complexity. Tomato 536 mutants with reduced GA levels or responses generally sustained pleiotropic effects, 537 which made it difficult to interpret its effect on leaf formation (Koornneef et al., 1990; 538 Jasinski et al., 2008; Yanai et al., 2011; Livne et al., 2015; Tomlinson et al., 2019). 539 Besides, specific reducing endogenous GA levels in leaf by overexpressing GA 540 deactivated gene GA2ox under the driving of leaf-specific promoter $p F I L$ resulted in 541 more complex leaf pattern, while was moderate compared to the substantial 542 simplification effect caused by increasing GA levels or responses (Shwartz et al., 543 2016).

544 BR and GA are known as two principal groups of plant growth-promoting 545 phytohormones for their functions in promoting cell elongation and differentiation. 
546 Defects in either of their biosynthesis or signaling transduction could result in 547 inhibited plant growth and dwarfism (Schwechheimer, 2011; Fridman and 548 Savaldi-Goldstein, 2013). The overlapping functions of BR and GA implied the 549 potential crosstalk between them, which attracted extensive attentions in the recent 550 one decade ( $\mathrm{Li}$ and $\mathrm{He}, 2013$ ). A breakthrough was the signaling interaction between 551 these two hormones reported by three different laboratories at the same time. In this 552 signaling model, the negative regulator of GA signaling, DELLAs, can physically 553 interact with BES1 and BZR1, the positive regulators of BR signaling (Bai et al., 554 2012; Gallego-Bartolomé et al., 2012; Li et al., 2012). Specifically, DELLAs only 555 interacted with the dephosphorylated active BZR1 and further inhibited BZR1-DNA 556 binding ability (Bai et al., 2012). This DELLA-BES1/BZR1 interaction also existed in 557 our research that tomato DELLA protein can physically bind to S1BES1.8 to interfere 558 with its function by abolishing SIBES1.8-DNA binding (Fig. 7). Later, the synthesis 559 model was proposed based on the results obtained from Arabidopsis and rice that BR 560 regulated the biosynthesis of GA. In Arabidopsis, the expressions of multiple GA 561 biosynthesis genes GA20oxs and GA3oxs were impaired in BR mutants. 562 Correspondingly, the bioactive GA contents were reduced, which was partly 563 responsible for the growth defects of BR mutants because external GA application or 564 expression of GA20oxl partially restored the defects. Furthermore, the direct 565 regulation of BES1 to the promoters of GA20oxl, GA3oxl and GA3ox4 were verified 566 (Unterholzner et al., 2015). Similarly, BZR1 directly bound to the promoters of 567 GA20ox2, GA3ox2 and GA2ox3 to regulate their expression in rice (Tong et al., 2014). 568 In our study, we determined the direct repression of SIBES1.8 to SlGA2ox2 and 569 SlGA2ox6, which led to the increase of bioactive GA contents that resulted in a 570 similar phenotype to increased GA level or response. Taken together, our results 571 obtained from tomato, in a certain extent, expanded our knowledge to BR and GA 572 crosstalk both in signaling level and biosynthesis level, and more efforts need to be 573 made in elaborating the crosstalk of these two hormones in different species.

574 As the key regulators of BR signaling, BES1 genes involved in numerous biological 
575 processes by directly interacting with key elements from other pathways. The 576 functional identification of BESI family genes were widely investigated in 577 Arabidopsis and other species, with critical roles in regulating cell elongation and 578 division (Xie et al., 2011), stress tolerance (Nolan et al., 2017), immune signaling 579 (Kang et al., 2015), meristem differentiation (Kondo et al., 2014) and male sterility 580 (Chen et al., 2019). Particularly, in tomato, the functional investigations of this family 581 were mainly focused on the two key members, SIBES1 and SIBZR1. With convincing 582 evidences, SIBES1 and SIBZR1 were verified to play important roles in regulating 583 heat stress tolerance (Yin et al., 2018), mediating autophagosome formation (Wang et 584 al., 2019), affecting pollen development (Yan et al., 2020), influencing wood 585 formation (Lee et al., 2021), releasing apical dominance (Xia et al., 2021) and 586 promoting fruit softening (Liu et al., 2021), while little is known about the functions 587 of other members. Our findings that SIBES1.8 greatly influences tomato leaf 588 morphogenesis obviously enriched the functional diversity of tomato BES1 family, 589 and more functional analyses of this family members in tomato and other species 590 would be benefit to understand the crosstalk of BR with other pathways in regulating 591 plant growth and stress responses.

592

\section{Supplementary data}

594 Supplementary data are available at $J X B$ online.

595 Fig. S1. Relative mRNA level of SIBES1.8 in over-expressed lines.

596 Fig. S2. Phenotype of one-month-old WT and OE-SlBES1.8 plants in Ailsa Craig 597 background.

598 Fig. S3. Knockout of SlBES1.8 didn't influence the normal development of tomato 599 leaf.

600 Fig. S4. Overexpression of SIBES1.8 resulted in similar consequence as the 601 exogenous application of $\mathrm{GA}_{3}$ in leaf morphogenesis.

602 Fig. S5. Expression pattern of genes involved in GA deactivation and signaling in WT 603 and OE-SIBES1.8 leaves in response to $\mathrm{GA}_{3}$ application. 
604 Fig. S6. Amino acids alignment of tomato and Arabidopsis DELLA proteins by 605 ClustalX.

606 Fig. S7. The PEST domain of SIBES1.8 and DELLA motif of SIDELLA are critical 607 for their interaction.

608 Table S1. Primers used in this study.

609 Table S2. GAs contents in WT and OE-SIBES1.8 leaves.

610 Table S3. DEGs after $\mathrm{GA}_{3}$ treatment in WT leaves.

611 Table S4. DEGs resulted by OE-SIBES1.8.

612 Table S5. 42 DEGs influenced by both $\mathrm{GA}_{3}$ and OE-SIBES1.8.

613

614 Acknowledgements

615 We would like to thank Analytical and Testing Center of Chongqing University for 616 providing laser scanning confocal microscope analysis. This work was supported by 617 the Fundamental Research Funds for the Central Universities (No. 618 2021CDJZYJH-002) and the National Natural Science Foundation of China (No. $61931972470,31772370,32002100)$.

620

621 Author contributions

622 Z. L. supervised the research; Z. L., Z. X. and D. S. designed the experiments; D. S., 623 W. X., Q. L. and L. W. performed the experiments; Y. L. provided experimental 624 assistance; D. S. wrote the manuscript and Z. X., Y. L. and Y. S. revised the paper. All 625 authors had read and approved the final manuscript.

626

627 Conflicts of interest

628 The authors declare no conflict of interest.

629

630

Data availability

631 All data supporting the results of this study are included in the paper and its 632 supplementary files. 


\section{References}

Alvarez JP, Furumizu C, Efroni I, Eshed Y, Bowman JL. 2016. Active suppression of a leaf meristem orchestrates determinate leaf growth. Elife $\mathbf{5}$.

Bai MY, Shang JX, Oh E, Fan M, Bai Y, Zentella R, Sun TP, Wang ZY. 2012. Brassinosteroid, gibberellin and phytochrome impinge on a common transcription module in Arabidopsis. Nature Cell Biology 14: 810-7.

Byrne ME. 2012. Making leaves. Current Topics in Developmental Biology 15: 24-30.

Byrne ME, Barley R, Curtis M, Arroyo JM, Dunham M, Hudson A, Martienssen RA. 2000. Asymmetric leaves1 mediates leaf patterning and stem cell function in Arabidopsis. Nature 408: 967-71.

Capua Y, Eshed Y. 2017. Coordination of auxin-triggered leaf initiation by tomato LEAFLESS. Proceedings of the National Academy of Sciences, USA 114: 3246-3251.

Chen H, Zou Y, Shang Y, Lin H, Wang Y, Cai R, Tang X, Zhou JM. 2008. Firefly luciferase complementation imaging assay for protein-protein interactions in plants. Plant Physiology 146: 368-76.

Chen LG, Gao Z, Zhao Z, Liu X, Li Y, Zhang Y, Liu X, Sun Y, Tang W. 2019. BZR1 Family Transcription Factors Function Redundantly and Indispensably in BR Signaling but Exhibit BRI1-Independent Function in Regulating Anther Development in Arabidopsis. Molecular Plant 12: 1408-1415.

Du F, Guan C, Jiao Y. 2018. Molecular Mechanisms of Leaf Morphogenesis. Molecular Plant 11: 1117-1134.

Efroni I, Eshed Y, Lifschitz E. 2010. Morphogenesis of simple and compound leaves: a critical review. The Plant Cell 22: 1019-32.

Fridman Y, Savaldi-Goldstein S. 2013. Brassinosteroids in growth control: how, when and where. Plant Science 209: 24-31.

Gallego-Bartolomé J, Minguet EG, Grau-Enguix F, Abbas M, Locascio A, Thomas SG, Alabadí D, Blázquez MA. 2012. Molecular mechanism for the 
interaction between gibberellin and brassinosteroid signaling pathways in Arabidopsis. Proceedings of the National Academy of Sciences, USA 109: 13446-51.

Goliber T, Kessler S, Chen JJ, Bharathan G, Sinha N. 1999. Genetic, molecular, and morphological analysis of compound leaf development. Current Topics in Developmental Biology 43: 259-90.

Grove MD, Spencer GF, Rohwedder WK, Mandava N, Worley JF, Warthen JD, Steffens GL, Flippen-Anderson JL, Cook JC. 1979. Brassinolide, a plant growth-promoting steroid isolated from Brassica napus pollen. Nature 281: 216-217.

He JX, Gendron JM, Sun Y, Gampala SS, Gendron N, Sun CQ, Wang ZY. 2005. BZR1 is a transcriptional repressor with dual roles in brassinosteroid homeostasis and growth responses. Science 307: 1634-8.

Hellens RP, Allan AC, Friel EN, Bolitho K, Grafton K, Templeton MD, Karunairetnam S, Gleave AP, Laing WA. 2005. Transient expression vectors for functional genomics, quantification of promoter activity and RNA silencing in plants. Plant Methods 1: 13.

Hirano K, Kouketu E, Katoh H, Aya K, Ueguchi-Tanaka M, Matsuoka M. 2012. The suppressive function of the rice DELLA protein SLR1 is dependent on its transcriptional activation activity. The Plant Journal 71: 443-53.

Israeli A, Ben-Herzel O, Burko Y, Shwartz I, Ben-Gera H, Harpaz-Saad S, Bar M, Efroni I, Ori N. 2021. Coordination of differentiation rate and local patterning in compound-leaf development. New Phytologist 229: 3558-3572.

Jasinski S, Tattersall A, Piazza P, Hay A, Martinez-Garcia JF, Schmitz G, Theres K, McCormick S, Tsiantis M. 2008. PROCERA encodes a DELLA protein that mediates control of dissected leaf form in tomato. The Plant Journal 56: 603-12.

Kang S, Yang F, Li L, Chen H, Chen S, Zhang J. 2015. The Arabidopsis transcription factor BRASSINOSTEROID INSENSITIVE1-ETHYL 
METHANESULFONATE-SUPPRESSOR1 is a direct substrate of MITOGEN-ACTIVATED PROTEIN KINASE6 and regulates immunity. Plant Physiology 167: 1076-86.

Kerstetter RA, Laudencia-Chingcuanco D, Smith LG, Hake S. 1997. Loss-of-function mutations in the maize homeobox gene, knotted1, are defective in shoot meristem maintenance. Development 124: 3045-54.

Kierzkowski D, Runions A, Vuolo F, et al. 2019. A Growth-Based Framework for Leaf Shape Development and Diversity. Cell 177: 1405-1418.e17.

Koka CV, Cerny RE, Gardner RG, Noguchi T, Fujioka S, Takatsuto S, Yoshida S, Clouse SD. 2000. A putative role for the tomato genes DUMPY and CURL-3 in brassinosteroid biosynthesis and response. Plant Physiology 122: 85-98.

Kondo Y, Ito T, Nakagami H, Hirakawa Y, Saito M, Tamaki T, Shirasu K, Fukuda H. 2014. Plant GSK3 proteins regulate xylem cell differentiation downstream of TDIF-TDR signalling. Nature Communications 5: 3504.

Koornneef M, Bosma TD, Hanhart CJ, van der Veen JH, Zeevaart JA. 1990. The isolation and characterization of gibberellin-deficient mutants in tomato. Theoretical and Applied Genetics 80: 852-7.

Lee J, Kim H, Park SG, et al. 2021. Brassinosteroid-BZR1/2-WAT1 module determines the high level of auxin signalling in vascular cambium during wood formation. New Phytologist 230: 1503-1516.

Li QF, He JX. 2013. Mechanisms of signaling crosstalk between brassinosteroids and gibberellins. Plant Signaling \& Behavior 8: e24686.

Li QF, Wang C, Jiang L, Li S, Sun SS, He JX. 2012. An interaction between BZR1 and DELLAs mediates direct signaling crosstalk between brassinosteroids and gibberellins in Arabidopsis. Science Signaling 5: ra72.

Liu H, Liu L, Liang D, et al. 2021. SlBES1 promotes tomato fruit softening through transcriptional inhibition of PMEU1. iScience 24: 102926.

Livne S, Lor VS, Nir I, Eliaz N, Aharoni A, Olszewski NE, Eshed Y, Weiss D. 2015. Uncovering DELLA-Independent Gibberellin Responses by 
Characterizing New Tomato procera Mutants. The Plant Cell 27: 1579-94.

Lodha M, Marco CF, Timmermans MC. 2013. The ASYMMETRIC LEAVES complex maintains repression of KNOX homeobox genes via direct recruitment of Polycomb-repressive complex2. Genes \& Development 27: 596-601.

Lombardi-Crestana S, da Silva Azevedo M, e Silva GF, Pino LE, Appezzato-da-Glória B, Figueira A, Nogueira FT, Peres LE. 2012. The tomato (Solanum lycopersicum cv. Micro-Tom) natural genetic variation Rg1 and the DELLA mutant procera control the competence necessary to form adventitious roots and shoots. Journal of Experimental Botany 63: 5689-703.

Luo Q, Lian HL, He SB, Li L, Jia KP, Yang HQ. 2014. COP1 and phyB Physically Interact with PIL1 to Regulate Its Stability and Photomorphogenic Development in Arabidopsis. The Plant Cell 26: 2441-2456.

Marín-de la Rosa N, Pfeiffer A, Hill K, et al. 2015. Genome Wide Binding Site Analysis Reveals Transcriptional Coactivation of Cytokinin-Responsive Genes by DELLA Proteins. PLoS Genetics 11: e1005337.

Martí E, Gisbert C, Bishop GJ, Dixon MS, García-Martínez JL. 2006. Genetic and physiological characterization of tomato cv. Micro-Tom. Journal of Experimental Botany 57: 2037-47.

Merelo P, Ram H, Pia Caggiano M, et al. 2016. Regulation of MIR165/166 by class II and class III homeodomain leucine zipper proteins establishes leaf polarity. Proceedings of the National Academy of Sciences, USA 113: 11973-11978.

Mitchell JW, Mandava N, Worley JF, Plimmer JR, Smith MV. 1970. Brassins--a new family of plant hormones from rape pollen. Nature 225: 1065-6.

Nolan T, Chen J, Yin Y. 2017. Cross-talk of Brassinosteroid signaling in controlling growth and stress responses. The Biochemical Journal 474: 2641-2661.

Ori N, Cohen AR, Etzioni A, et al. 2007. Regulation of LANCEOLATE by miR319 is required for compound-leaf development in tomato. Nature Genetics 39: 787-91. 
Reinhardt D, Mandel T, Kuhlemeier C. 2000. Auxin regulates the initiation and radial position of plant lateral organs. The Plant Cell 12: 507-18.

Reinhardt D, Pesce ER, Stieger P, Mandel T, Baltensperger K, Bennett M, Traas J, Friml J, Kuhlemeier C. 2003. Regulation of phyllotaxis by polar auxin transport. Nature 426: 255-60.

Rodriguez RE, Mecchia MA, Debernardi JM, Schommer C, Weigel D, Palatnik JF. 2010. Control of cell proliferation in Arabidopsis thaliana by microRNA miR396. Development 137: 103-12.

Schwechheimer C. 2011. Gibberellin signaling in plants - the extended version. Frontiers in Plant Science 2: 107.

Shwartz I, Levy M, Ori N, Bar M. 2016. Hormones in tomato leaf development. Developmental Biology 419: 132-142.

Su D, Xiang W, Wen L, Lu W, Shi Y, Liu Y, Li Z. 2021. Genome-wide identification, characterization and expression analysis of BES1 gene family in tomato. BMC Plant Biology 21: 161.

Tatematsu K, Toyokura K, Miyashima S, Nakajima K, Okada K. 2015. A molecular mechanism that confines the activity pattern of miR165 in Arabidopsis leaf primordia. The Plant Journal 82: 596-608.

Tomlinson L, Yang Y, Emenecker R, Smoker M, Taylor J, Perkins S, Smith J, MacLean D, Olszewski NE, Jones JDG. 2019. Using CRISPR/Cas9 genome editing in tomato to create a gibberellin-responsive dominant dwarf DELLA allele. Plant Biotechnology Journal 17: 132-140.

Tong H, Xiao Y, Liu D, Gao S, Liu L, Yin Y, Jin Y, Qian Q, Chu C. 2014. Brassinosteroid regulates cell elongation by modulating gibberellin metabolism in rice. The Plant Cell 26: 4376-93.

Tsukaya H. 2018. Leaf shape diversity with an emphasis on leaf contour variation, developmental background, and adaptation. Seminars in Cell \& Developmental Biology 79: 48-57.

Unterholzner SJ, Rozhon W, Papacek M, Ciomas J, Lange T, Kugler KG, Mayer 
KF, Sieberer T, Poppenberger B. 2015. Brassinosteroids Are Master Regulators of Gibberellin Biosynthesis in Arabidopsis. The Plant Cell 27: 2261-72.

Vlad D, Kierzkowski D, Rast MI, et al. 2014. Leaf shape evolution through duplication, regulatory diversification, and loss of a homeobox gene. Science 343: 780-3.

Wang Y, Cao JJ, Wang KX, Xia XJ, Shi K, Zhou YH, Yu JQ, Zhou J. 2019. BZR1

Mediates Brassinosteroid-Induced Autophagy and Nitrogen Starvation in Tomato. Plant Physiology 179: 671-685.

Xia X, Dong H, Yin Y, et al. 2021. Brassinosteroid signaling integrates multiple pathways to release apical dominance in tomato. Proceedings of the National Academy of Sciences, USA 118.

Xie L, Yang C, Wang X. 2011. Brassinosteroids can regulate cellulose biosynthesis by controlling the expression of CESA genes in Arabidopsis. Journal of Experimental Botany 62: 4495-506.

Yan MY, Xie DL, Cao JJ, Xia XJ, Shi K, Zhou YH, Zhou J, Foyer CH, Yu JQ. 2020. Brassinosteroid-mediated reactive oxygen species are essential for tapetum degradation and pollen fertility in tomato. The Plant Journal 102: 931-947.

Yanai O, Shani E, Russ D, Ori N. 2011. Gibberellin partly mediates LANCEOLATE activity in tomato. The Plant Journal 68: 571-82.

Yin Y, Qin K, Song X, Zhang Q, Zhou Y, Xia X, Yu J. 2018. BZR1 Transcription Factor Regulates Heat Stress Tolerance Through FERONIA Receptor-Like Kinase-Mediated Reactive Oxygen Species Signaling in Tomato. Plant \& Cell Physiology 59: 2239-2254.

Yin Y, Vafeados D, Tao Y, Yoshida S, Asami T, Chory J. 2005. A new class of transcription factors mediates brassinosteroid-regulated gene expression in Arabidopsis. Cell 120: 249-59.

Yoshida H, Hirano K, Sato T, et al. 2014. DELLA protein functions as a 
transcriptional activator through the DNA binding of the indeterminate domain family proteins. Proceedings of the National Academy of Sciences, USA 111: 7861-6.

Yu X, Li L, Li L, Guo M, Chory J, Yin Y. 2008. Modulation of brassinosteroid-regulated gene expression by Jumonji domain-containing proteins ELF6 and REF6 in Arabidopsis. Proceedings of the National Academy of Sciences, USA 105: 7618-23.

Zhiponova MK, Vanhoutte I, Boudolf V, et al. 2013. Brassinosteroid production and signaling differentially control cell division and expansion in the leaf. New Phytologist 197: 490-502. 


\section{Figure legends}

Fig. 1. Overexpression of SIBES1.8 resulted in changed morphogenesis of tomato leaf. (A, B) The mature leaf at 35 DPG and leaf primordium at 15 DPG of WT (A) and OE-SlBES1.8 (B) plants in micro-tom background. TL, terminal leaflet; LL, lateral leaflet; LLP, lateral leaflet primordium; Se, serration; DPG, days post germination; bar in mature leaf indicates $1 \mathrm{~cm}$, bar in leaf primordium indicates 100 $\mu \mathrm{m}$. (C) Serration numbers of terminal leaflet and lateral leaflet in mature leaves. At least 20 leaves were counted for each column. (D) Leaves of wild type and OE-SIBES1.8 plants in Ailsa Craig background. L, leaf; bar $=2 \mathrm{~cm}$. (E) Silhouettes of the seventh leaf from wild type and OE-SIBES1.8 plants (Ailsa Craig background) at 45 DPG. 1st indicates terminal and primary lateral leaflets; 2nd indicates secondary lateral leaflets; 3rd indicates intercalary leaflets; bar $=2 \mathrm{~cm}$. (F) Leaflet numbers of wild type and OE-SIBES1.8 plants presented in (D). At least 20 leaves were counted for each column. $* *$ and $* * *$ refer to significant differences between WT and OE-SlBES1.8 lines with $P<0.01$ and $P<0.001$ respectively (two-tailed Student's $t$-test).

Fig. 2. Overexpression of SlBES1.8 resulted in similar consequence as the exogenous application of $\mathrm{GA}_{3}$ in leaf morphogenesis. (A) Leaf phenotype of WT seedlings at 16 DPG without $\mathrm{GA}_{3}$ application. (B) Leaf phenotype of WT seedlings at 16 DPG with $50 \mu \mathrm{M} \mathrm{GA}_{3}$ application. (C) Leaf phenotype of OE-SlBES1.8 seedlings at $16 \mathrm{DPG}$ without $\mathrm{GA}_{3}$ application. Arrowhead indicates the serration of leaf. Blue and red circles indicate lateral leaflet (LL) and lateral leaflet primordium (LLP) respectively. L, leaf; white bar $=1 \mathrm{~cm}$; black bar $=1 \mathrm{~mm}$.

Fig. 3. Overexpression of $S I B E S 1.8$ reduced the sensitivity to $\mathrm{GA}_{3}$ while increased the sensitivity to paclobutrazol (PAC). (A) Potential cis-elements in $2 \mathrm{~kb}$ upstream promoter of SlBES1.8. The GA responsive element TATC-box is highlighted with red font. (B) The expression pattern of SIBES1.8 in response to $\mathrm{GA}_{3}$ application. 
Seedlings were soaked into MS/2 medium containing $50 \mu \mathrm{M} \mathrm{GA}$ for 1, 2, 4, 8 and 16 hours. Values are means \pm SD of three biological replicates. (C) Phenotype of WT and OE-SlBES1.8 seedlings at $10 \mathrm{DPG}$ with or without $50 \mu \mathrm{M} \mathrm{GA} 3$ application. Arrow indicates the elongated shoot. Bar $=1 \mathrm{~cm}$. $(\mathrm{D}, \mathrm{E})$ Length of primary root $(\mathrm{D})$ and first shoot (E) of seedlings with or without $\mathrm{GA}_{3}$ application $(10 \mu \mathrm{M}$ and $50 \mu \mathrm{M})$. At least 20 seedlings were counted for each column. (F) Phenotype of wild type and OE-SlBES1.8 plants and corresponding leaves with or without $\mathrm{GA}_{3}(100 \mu \mathrm{M})$ or PAC $(50 \mu \mathrm{M})$ application. Bar $=1 \mathrm{~cm} . * * *$ and $* * *$ refer to significant differences with $P$ $<0.05, P<0.01$ and $P<0.001$ respectively (two-tailed Student's $t$-test).

Fig. 4. Endogenous gibberellin (GA) contents in WT and OE-SlBES1.8 leaves. (A) Part of generalized scheme of GA biosynthesis and deactivation in higher plants. Serial numbers indicate those GAs that have been detected in our study. 2ox, GA 2-oxidase; 3ox, GA 3-oxidase; 13ox, GA 13-oxidase; 20ox, GA 20-oxidase. (B) Contents of bioactive and inactive GAs detected in our study. (C) Total GAs contents in WT and OE-SIBES1.8 leaves. (D) Proportion of two richest GAs $\left(\mathrm{GA}_{3}\right.$ and $\left.\mathrm{GA}_{8}\right)$ in WT and OE-SlBES1.8 leaves. (E) Proportion of another two bioactive GAs $\left(\mathrm{GA}_{1}\right.$ and $\left.\mathrm{GA}_{4}\right)$ in WT and OE-SlBES1.8 leaves. Values are means \pm SD of three biological replicates. * refers to significant differences between WT and OE-SIBES1.8 line with $P<0.05$ (two-tailed Student's $t$-test).

\section{Fig. 5. Transcriptome analysis of WT and OE-SIBES1.8 leaves with or without}

GA $_{3}$ application. (A) Numbers of up- or down-regulated differentially expressed genes (DEGs, FC $>2, Q<0.05$ ) in distinct comparisons. (B) Venn diagram showing the overlap between sets of DEGs influenced by $\mathrm{GA}_{3}$ treatment in WT (WT GA 3 / WT Mock) or by overexpression of SlBES1.8 (OE Mock / WT Mock). (C) Heat map showing the expression profiles of 42 common DEGs in (B). Group $\square$ containing DEGs that are both up- or down-regulated by $\mathrm{GA}_{3}$ and OE-SIBES1.8. Group containing DEGs that are regulated by $\mathrm{GA}_{3}$ and OE-SIBES1.8 with opposite trends. 
(D) Expression comparison of those 42 common DEGs after $\mathrm{GA}_{3}$ application in WT and OE-SlBES1.8 leaves. Group $\square$ and $\square$ containing DEGs with smaller or bigger effect respectively by $\mathrm{GA}_{3}$ application in OE-SIBES1.8 leaves compared with WT. FC, fold change.

Fig. 6. SIBES1.8 directly represses the expression of SlGA2ox2, SIGA2ox6 and SIGIDIb-1 and promotes the degradation of SIDELLA. (A-C) Relative expression levels of SlGA2ox2 (A), SlGA2ox6 (B) and SlGID1b-1 (C) in response to GA3 application in WT and OE-SlBES1.8 lines. Values are means \pm SD of three biological replicates. Different letters indicate significant differences $(P<0.05)$ according to one-way ANOVA test. (D) Diagram showing the promoter fragments of SlGA2ox2, SlGA2ox6 and SlGIDIb-1 used in electrophoretic mobility shift assay (EMSA, between orange lines), yeast one-hybrid assays ( $\mathrm{Y} 1 \mathrm{H}$, between green lines) and dual-luciferase assays (between blue lines). The position of G-box (CACGTG) is indicated by red line. (E) EMSA showing the binding of SIBES1.8 to the promoters of SlGA2ox2, SlGA2ox6 and SlGIDIb-1. GST alone incubated with biotin probe was used as negative control. Mutant biotin probe and competitor probe confirmed the specific binding. ++, increased amount of competitor probes. (F) Y1H showing the binding of SIBES1.8 to the promoters of SlGA2ox2, SlGA2ox6 and SlGIDIb-1. Yeast transformants were cultured on SD/-Leu or SD/-Leu/+AbA mediums for 3 days. pGADT7-empty plasmid was used as control. The screening concentration of AbA was indicated in the bottom. (G) Diagram of reporters and effector used in dual-luciferase assays. Promoters of SlGA2ox2, SlGA2ox6 and SlGID1b-1 were constructed into reporter, and full length coding sequence of SIBES1.8 was constructed into effector. $(\mathrm{H})$ Results of dual-luciferase assays showing the inhibition of SIBES1.8 to the promoter activity of SIGA2ox2, SlGA2ox6 and SlGIDIb-1. The LUC/REN ratio of the group of empty effector was regarded as calibrator (set as 1). At least six biological replicates were used for each column. $* *$ and $* * *$ refer to significant differences between empty and SIBES1.8 effectors with $P<0.01$ and $P<$ 
0.001 respectively (two-tailed Student's $t$-test). (I) Immunoblots showed the content of SIDELLA. $\mathrm{GA}_{3}$ promoted the degradation whereas PAC promoted the accumulation of SIDELLA, as expected. Actin is used as a loading control.

Fig. 7. SIDELLA interacts with SIBES1.8 and suppresses its transcriptional repression ability by inhibiting SIBES1.8-DNA binding. (A) Yeast two-hybrid $(\mathrm{Y} 2 \mathrm{H})$ assay for interaction between SIDELLA and S1BES1.8 proteins. AD-T plus BD-53 or BD-Lam were used as the positive and negative control respectively. DDO, SD/-Leu/-Trp medium; QDO, SD/-Ade/-His/-Leu/-Trp medium. (B) Bimolecular fluorescence complementation (BiFC) assay in tobacco leaves for interaction between S1BES1.8 and SIDELLA proteins. Bar $=50 \mu \mathrm{m}$. (C) Firefly luciferase complementation imaging (LCI) assay in tobacco leaves for interaction between SIBES1.8 and SIDELLA proteins. (D) Diagram of reporter and effectors used in dual-luciferase assays. SIDELLA and SIBES1.8 were fused with GAL4BD to generate the effector constructs. VP16 was used as the transcriptional activation control. (E) Suppression of SIDELLA on the transcriptional repression activity of SIBES1.8 detecting by GAL4-responsive reporter system in tobacco leaves. Reporter was co-expressed with SIBES1.8 or SIDELLA effector alone or together in tobacco leaves. The LUC/REN ratio of the group of empty effector was regarded as calibrator (set as 1). At least six biological replicates were used for each column. The significant differences are indicated with letters $(P<0.05$, one-way ANOVA test). (F) EMSA showing the inhibition of SIDELLA on the binding of SIBES1.8 to the promoters of SlGA2ox2, SlGA2ox6 and SlGID1b-1. Biotin probes were incubated with GST-SIBES1.8 alone or together with GST-SIDELLA protein. ++ indicates increased amount of GST-SIDELLA proteins; m, mutant biotin probe.

Fig. 8. Proposed model depicting the molecular mechanism of SIBES1.8 in the regulation of tomato leaf morphogenesis. SIBES1.8 represses the transcriptions of SlGA2ox2 and SlGA2ox6, which encode GA deactivation enzymes, to positively 
regulate bioactive GA contents and SIDELLA degradation. Meanwhile, SIBES1.8 also represses the expression of GA receptor, $S I G I D 1 b$-1, leading to a feedback regulation in SIDELLA degradation. On the other hand, SIDELLA physically interacts with SIBES1.8 and inhibits its transcriptional regulation ability. The degradation of SIDELLA releases its inhibition to SIBES1.8, which further promotes SIDELLA degradation. By such mediation both in GA level and signaling, SIBES1.8 greatly influences tomato leaf morphogenesis. 


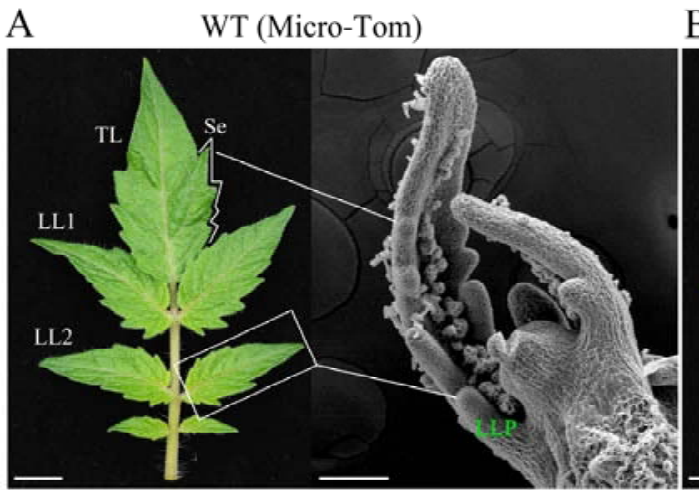

B OE-SIBES1.8

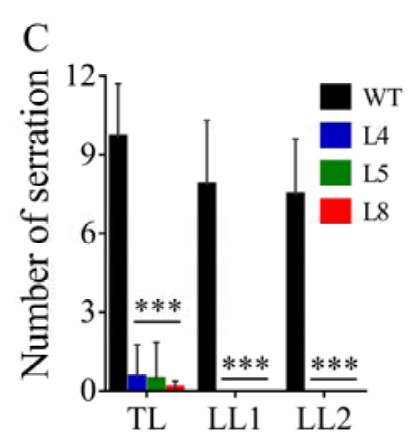

E WT (Ailsa Craig)
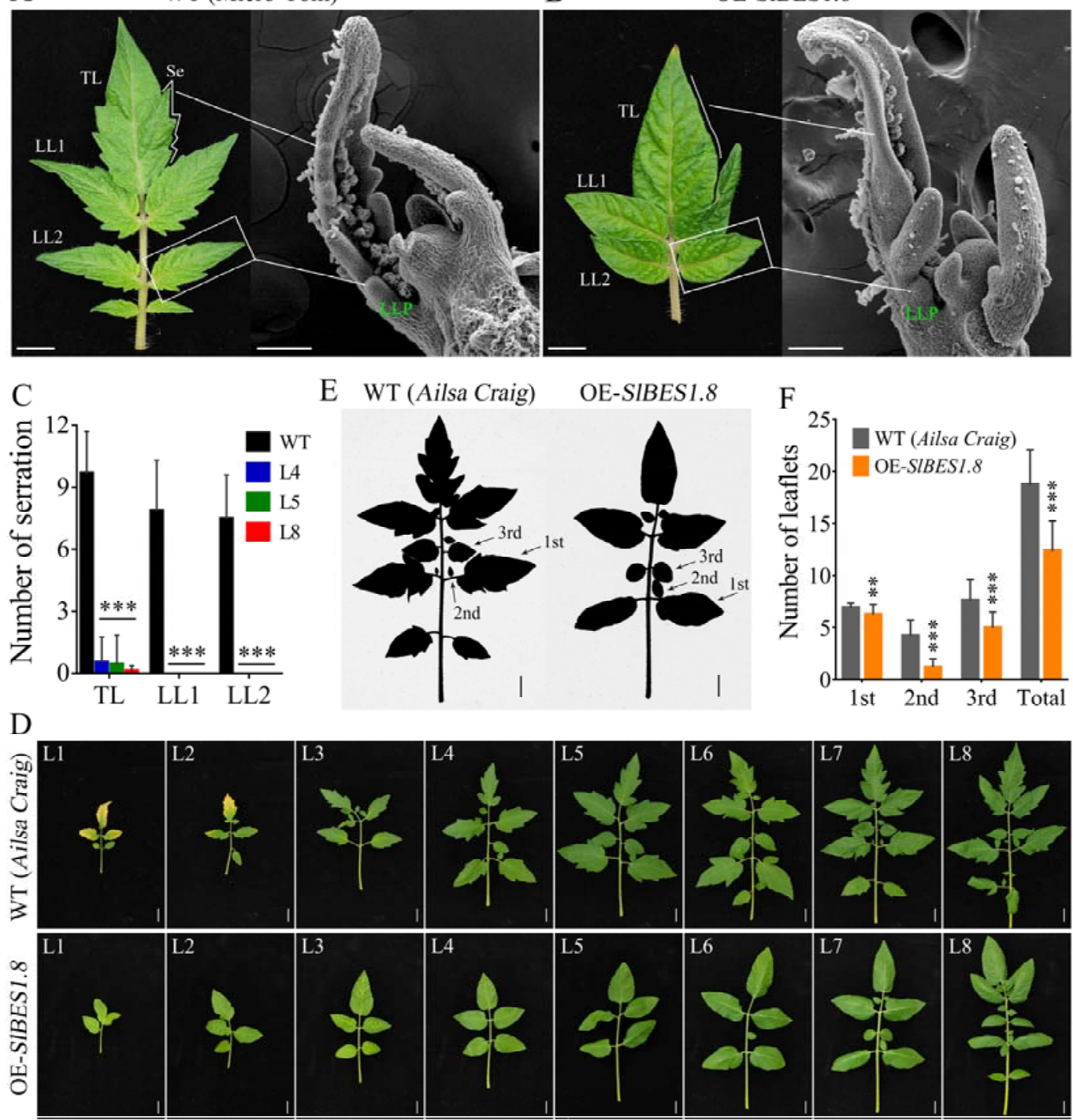

Fig. 1. Overexpression of SlBES1.8 resulted in changed morphogenesis of tomato leaf. (A, B) The mature leaf at 35 DPG and leaf primordium at 15 DPG of WT (A) and OE-SlBES1.8 (B) plants in micro-tom background. TL, terminal leaflet; LL, lateral leaflet; LLP, lateral leaflet primordium; Se, serration; DPG, days post germination; bar in mature leaf indicates $1 \mathrm{~cm}$, bar in leaf primordium indicates 100 $\mu \mathrm{m}$. (C) Serration numbers of terminal leaflet and lateral leaflet in mature leaves. At least 20 leaves were counted for each column. (D) Leaves of wild type and OE-SIBES1.8 plants in Ailsa Craig background. L, leaf; bar $=2 \mathrm{~cm}$. (E) Silhouettes of the seventh leaf from wild type and OE-SlBES1.8 plants (Ailsa Craig background) at 45 DPG. 1st indicates terminal and primary lateral leaflets; 2nd indicates secondary lateral leaflets; 3rd indicates intercalary leaflets; bar $=2 \mathrm{~cm}$. (F) Leaflet numbers of wild type and OE-SIBES1.8 plants presented in (D). At least 20 leaves were counted for each column. $* *$ and $* * *$ refer to significant differences between WT and OE-SlBES1.8 lines with $P<0.01$ and $P<0.001$ respectively (two-tailed Student's $t$-test). 
bioRxiv preprint doi: https://doi.org/10.1101/2021.12.10.472069; this version posted December 10,2021 . The copyright holder for this preprint (which was not certified by peer review) is the author/funder. All rights reserved. No reuse allowed without permission. 

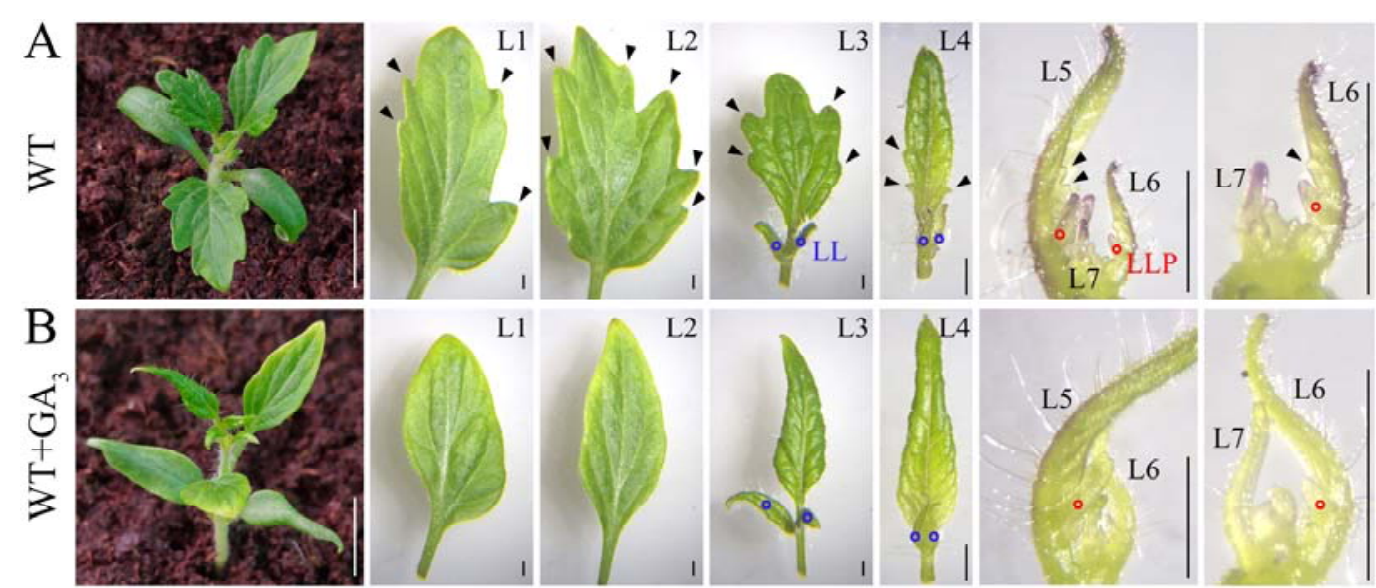

L1

L2

L3
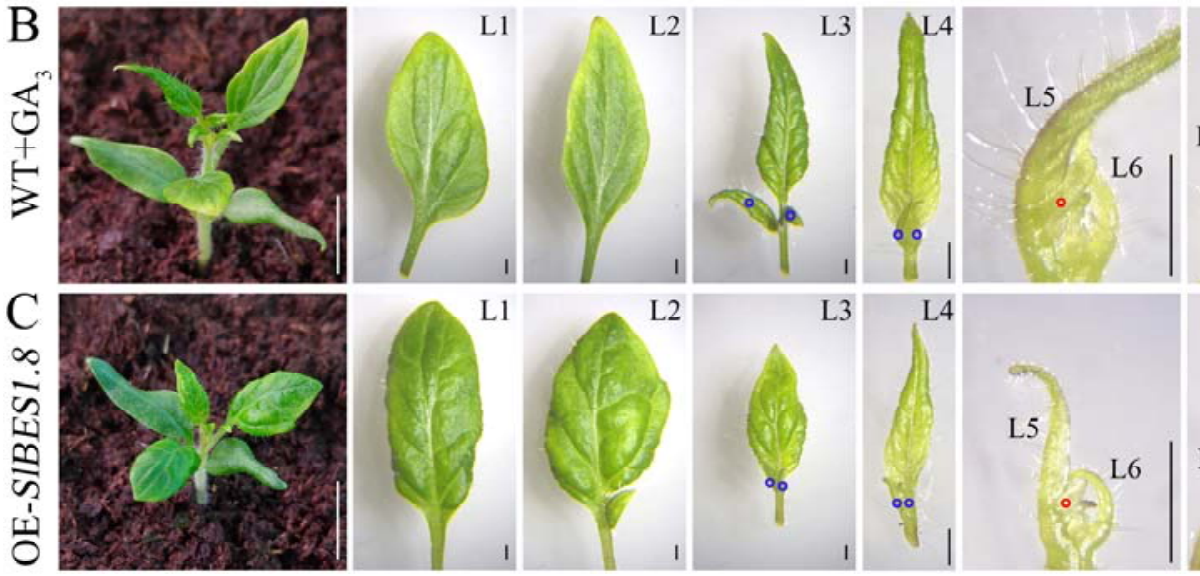

L6

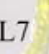

L6

L7

Fig. 2. Overexpression of SlBES1.8 resulted in similar consequence as the exogenous application of $\mathbf{G A}_{3}$ in leaf morphogenesis. (A) Leaf phenotype of WT seedlings at $16 \mathrm{DPG}$ without $\mathrm{GA}_{3}$ application. (B) Leaf phenotype of WT seedlings at 16 DPG with $50 \mu \mathrm{M} \mathrm{GA}_{3}$ application. (C) Leaf phenotype of OE-SlBES1.8 seedlings at 16 DPG without $\mathrm{GA}_{3}$ application. Arrowhead indicates the serration of leaf. Blue and red circles indicate lateral leaflet (LL) and lateral leaflet primordium (LLP) respectively. L, leaf; white bar $=1 \mathrm{~cm}$; black bar $=1 \mathrm{~mm}$. 
A

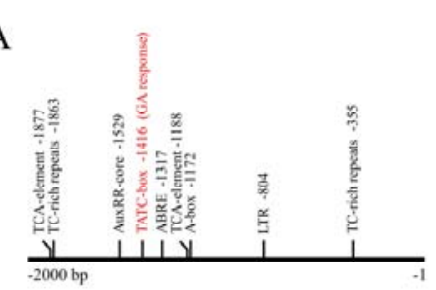

B

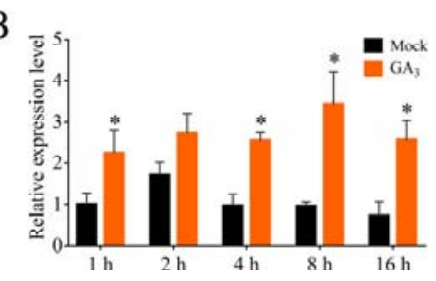

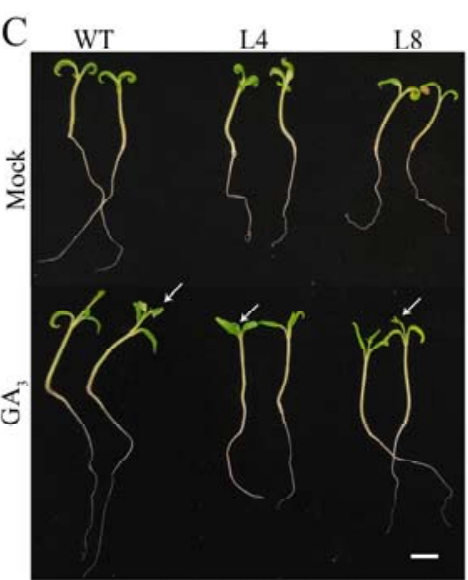

$\mathrm{GA}_{3}$

F Mock

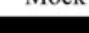

Mock
PAC

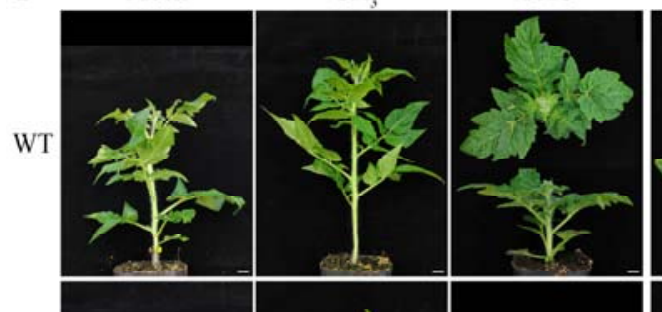

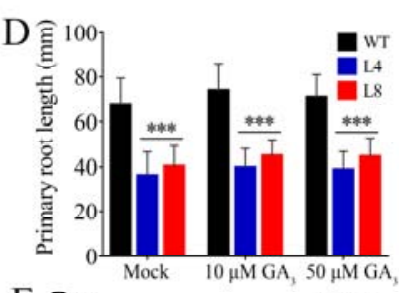

$\mathrm{E}$ 而

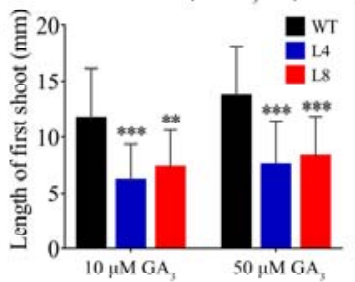

$\mathrm{GA}$

PAC
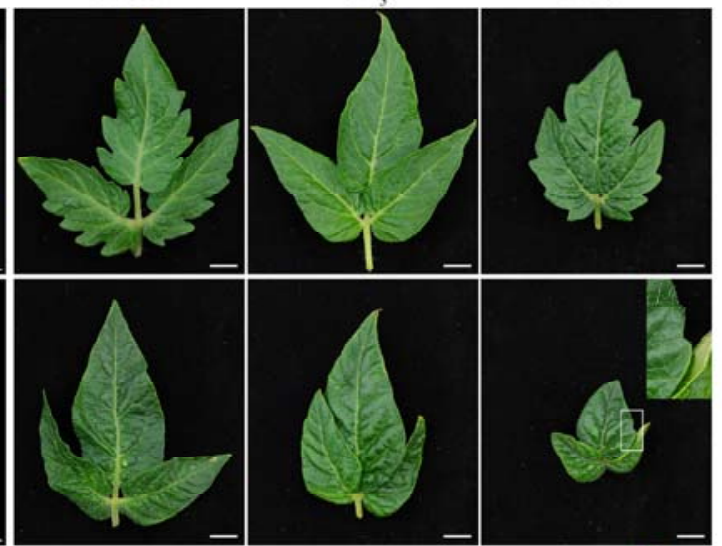

L4
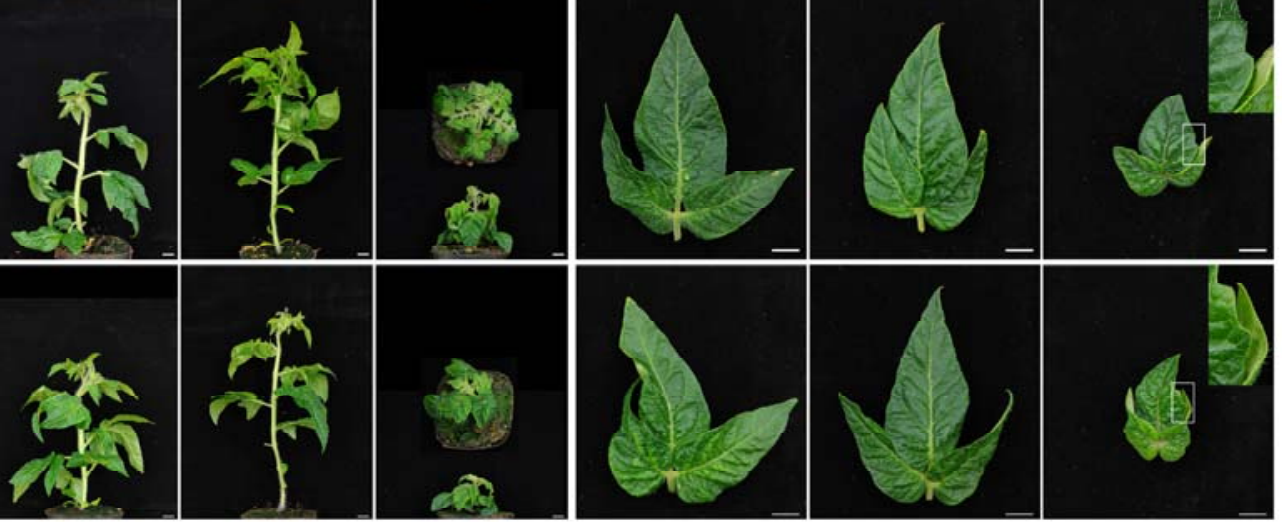

Fig. 3. Overexpression of SIBES1.8 reduced the sensitivity to $\mathrm{GA}_{3}$ while increased the sensitivity to paclobutrazol (PAC). (A) Potential cis-elements in $2 \mathrm{~kb}$ upstream promoter of SIBES1.8. The GA responsive element TATC-box is highlighted with red font. (B) The expression pattern of SlBES1.8 in response to $\mathrm{GA}_{3}$ application. Seedlings were soaked into MS/2 medium containing $50 \mu \mathrm{M} \mathrm{GA}_{3}$ for $1,2,4,8$ and 16 hours. Values are means \pm SD of three biological replicates. (C) Phenotype of WT and OE-SlBES1.8 seedlings at $10 \mathrm{DPG}$ with or without $50 \mu \mathrm{M} \mathrm{GA} 3$ application. Arrow indicates the elongated shoot. $B a r=1 \mathrm{~cm}$. $(D, E)$ Length of primary root $(D)$ and first shoot (E) of seedlings with or without $\mathrm{GA}_{3}$ application $(10 \mu \mathrm{M}$ and $50 \mu \mathrm{M})$. At least 20 seedlings were counted for each column. (F) Phenotype of wild type and OE-SlBES1.8 plants and corresponding leaves with or without $\mathrm{GA}_{3}(100 \mu \mathrm{M})$ or PAC $(50 \mu \mathrm{M})$ application. Bar $=1 \mathrm{~cm} . *, * *$ and $* * *$ refer to significant differences with $P$ $<0.05, P<0.01$ and $P<0.001$ respectively (two-tailed Student's $t$-test). 

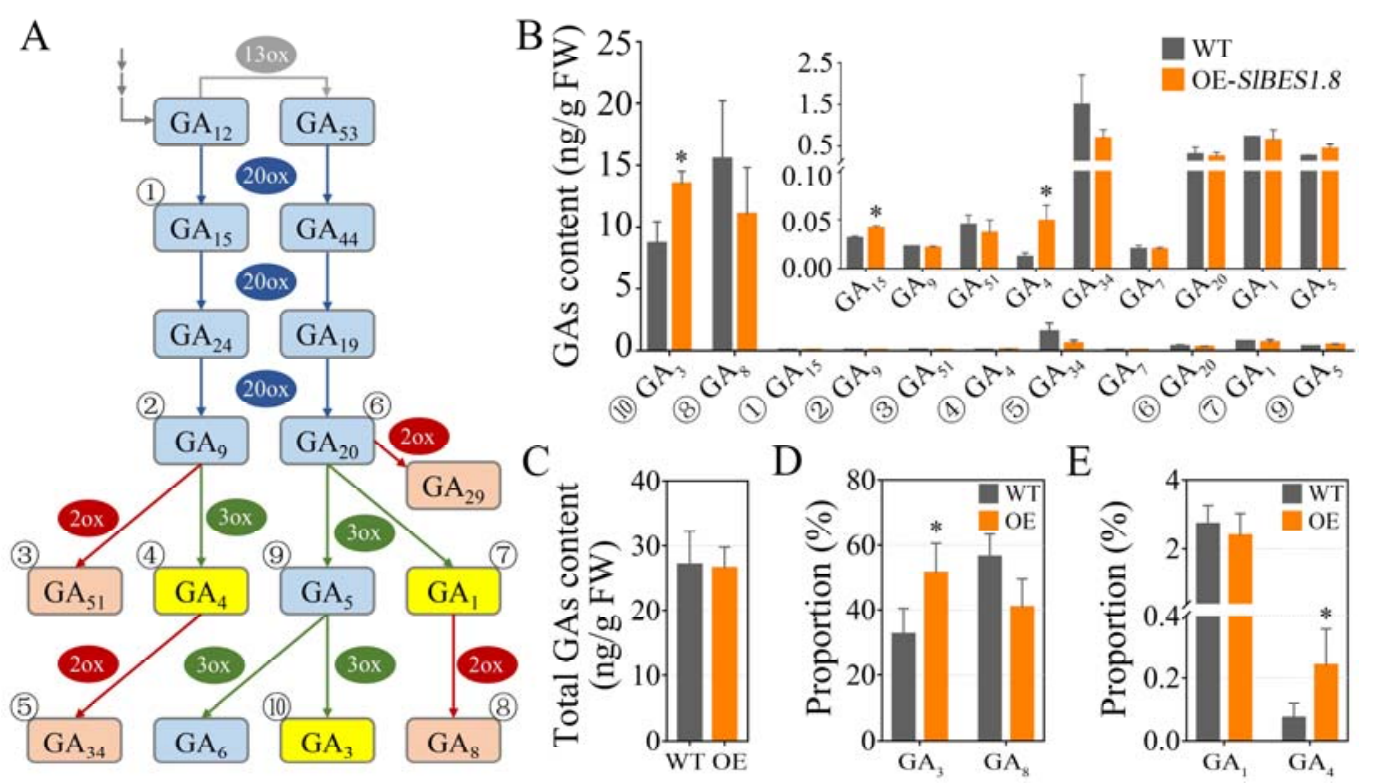

Fig. 4. Endogenous gibberellin (GA) contents in WT and OE-SlBES1.8 leaves. (A) Part of generalized scheme of GA biosynthesis and deactivation in higher plants. Serial numbers indicate those GAs that have been detected in our study. 2ox, GA 2-oxidase; 3ox, GA 3-oxidase; 13ox, GA 13-oxidase; 20ox, GA 20-oxidase. (B) Contents of bioactive and inactive GAs detected in our study. (C) Total GAs contents in WT and OE-SlBES1.8 leaves. (D) Proportion of two richest GAs $\left(\mathrm{GA}_{3}\right.$ and $\left.\mathrm{GA}_{8}\right)$ in WT and OE-SlBES1.8 leaves. (E) Proportion of another two bioactive GAs $\left(\mathrm{GA}_{1}\right.$ and $\mathrm{GA}_{4}$ ) in WT and OE-SlBES1.8 leaves. Values are means \pm SD of three biological replicates. * refers to significant differences between WT and OE-SlBES1.8 line with $P<0.05$ (two-tailed Student's $t$-test). 
A

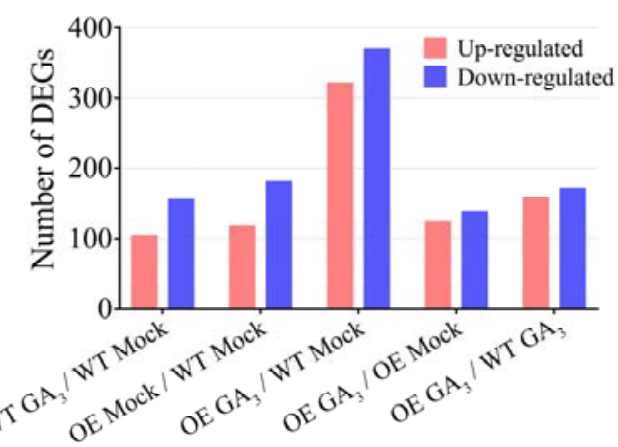

$\mathrm{C}$

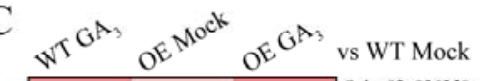

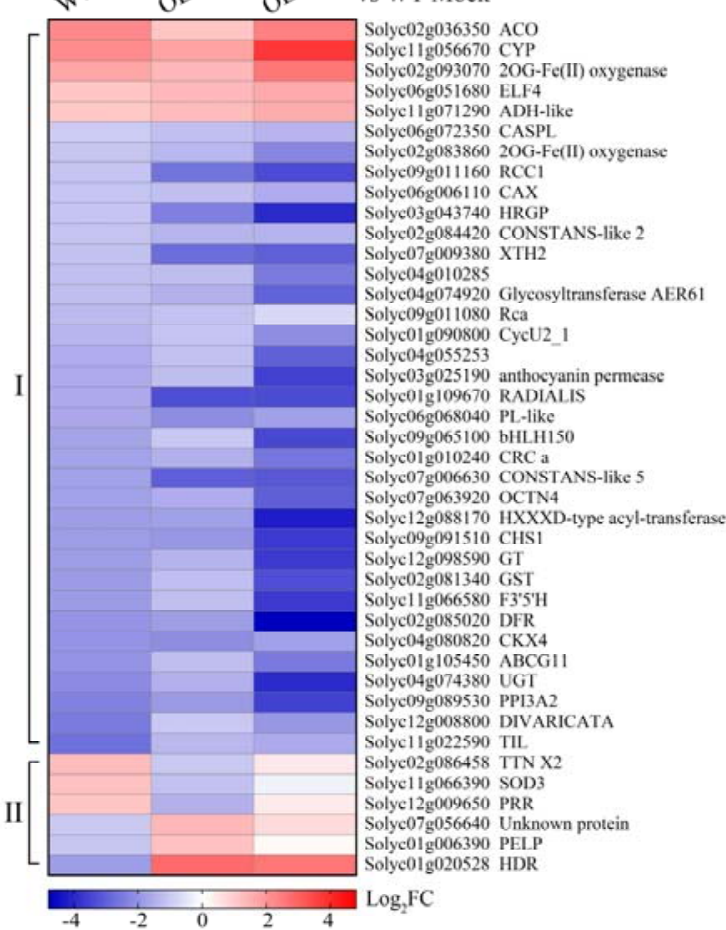

B

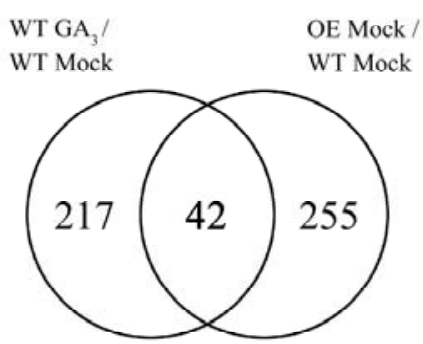

$\mathrm{D}$

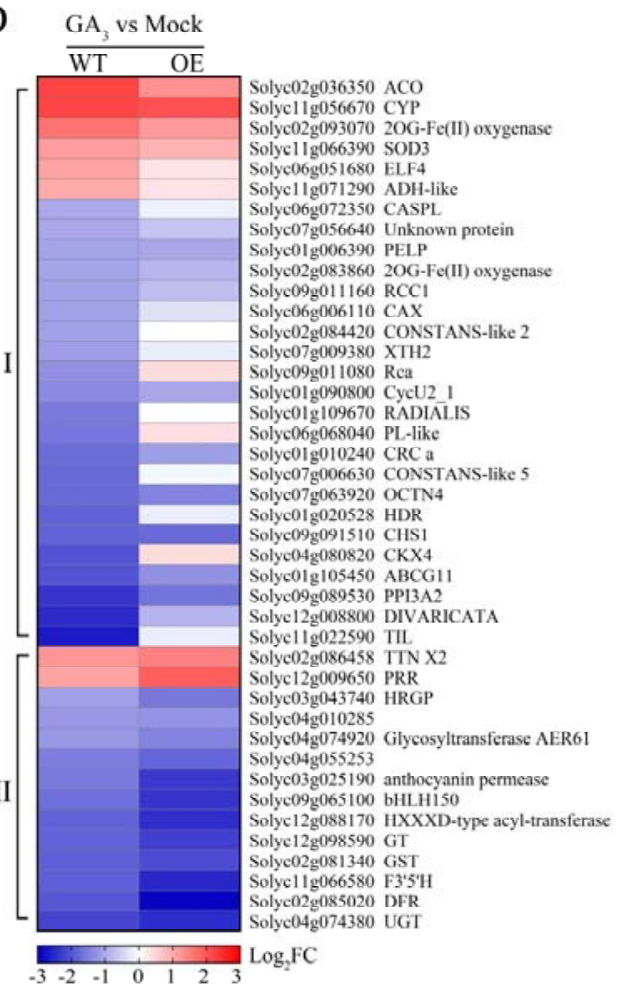

Fig. 5. Transcriptome analysis of WT and OE-SIBES1.8 leaves with or without $\mathbf{G A}_{3}$ application. (A) Numbers of up- or down-regulated differentially expressed genes (DEGs, FC $>2, Q<0.05$ ) in distinct comparisons. (B) Venn diagram showing

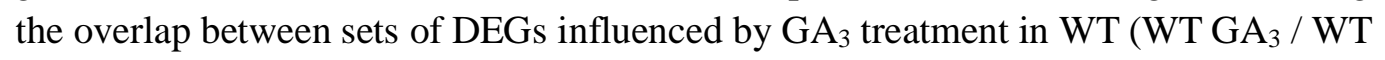
Mock) or by overexpression of SlBES1.8 (OE Mock / WT Mock). (C) Heat map showing the expression profiles of 42 common DEGs in (B). Group $\square$ containing DEGs that are both up- or down-regulated by $\mathrm{GA}_{3}$ and OE-SIBES1.8. Group $\square$ containing DEGs that are regulated by $\mathrm{GA}_{3}$ and OE-SIBES1.8 with opposite trends. (D) Expression comparison of those 42 common DEGs after $\mathrm{GA}_{3}$ application in WT and OE-SlBES1.8 leaves. Group $\square$ and $\square$ containing DEGs with smaller or bigger effect respectively by $\mathrm{GA}_{3}$ application in OE-SlBES1.8 leaves compared with WT. FC, fold change. 


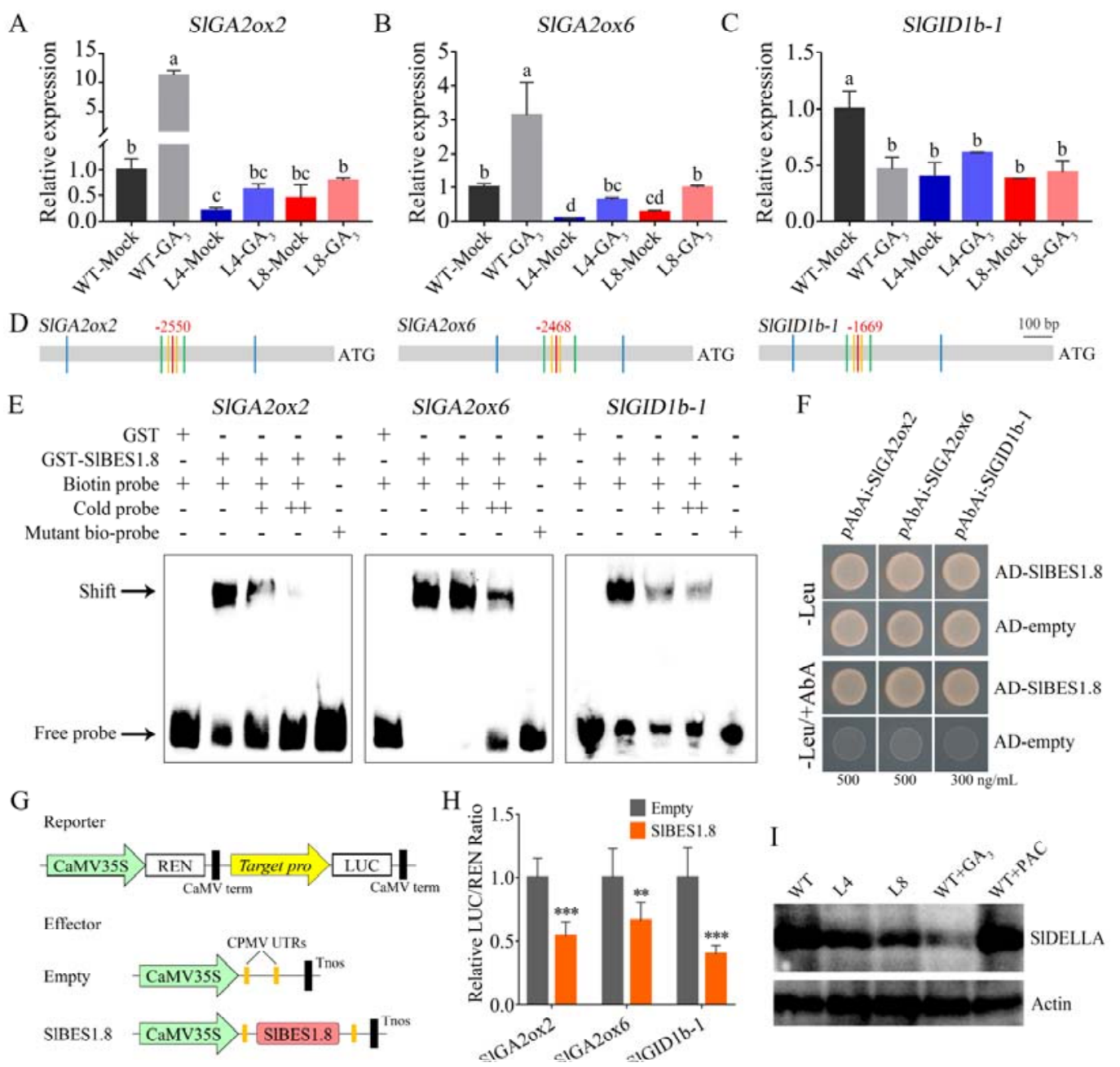

Fig. 6. SIBES1.8 directly represses the expression of SlGA2ox2, SlGA2ox6 and SIGIDIb-1 and promotes the degradation of SIDELLA. (A-C) Relative expression levels of SlGA2ox2 (A), SlGA2ox6 (B) and SlGIDIb-1 (C) in response to GA3 application in WT and OE-SlBES1.8 lines. Values are means \pm SD of three biological replicates. Different letters indicate significant differences $(P<0.05)$ according to one-way ANOVA test. (D) Diagram showing the promoter fragments of SlGA2ox2, SlGA2ox6 and SlGIDlb-1 used in electrophoretic mobility shift assay (EMSA, between orange lines), yeast one-hybrid assays ( $\mathrm{Y} 1 \mathrm{H}$, between green lines) and dual-luciferase assays (between blue lines). The position of G-box (CACGTG) is indicated by red line. (E) EMSA showing the binding of SIBES1.8 to the promoters of SlGA2ox2, SlGA2ox6 and SlGID1b-1. GST alone incubated with biotin probe was used as negative control. Mutant biotin probe and competitor probe confirmed the specific binding. ++, increased amount of competitor probes. (F) $\mathrm{Y} 1 \mathrm{H}$ showing the binding of SIBES1.8 to the promoters of SlGA2ox2, SlGA2ox6 and SlGID1b-1. Yeast transformants were cultured on SD/-Leu or SD/-Leu/+AbA mediums for 3 days. pGADT7-empty plasmid was used as control. The screening concentration of AbA was indicated in the bottom. (G) Diagram of reporters and effector used in dual-luciferase assays. Promoters of SlGA2ox2, SlGA2ox6 and SlGIDIb-1 were 
constructed into reporter, and full length coding sequence of SIBES1.8 was constructed into effector. $(\mathrm{H})$ Results of dual-luciferase assays showing the inhibition of SIBES1.8 to the promoter activity of SlGA2ox2, SlGA2ox6 and SlGIDIb-1. The LUC/REN ratio of the group of empty effector was regarded as calibrator (set as 1). At least six biological replicates were used for each column. $* *$ and $* * *$ refer to significant differences between empty and SIBES1.8 effectors with $P<0.01$ and $P<$ 0.001 respectively (two-tailed Student's $t$-test). (I) Immunoblots showed the content of SIDELLA. $\mathrm{GA}_{3}$ promoted the degradation whereas PAC promoted the accumulation of SIDELLA, as expected. Actin is used as a loading control. 

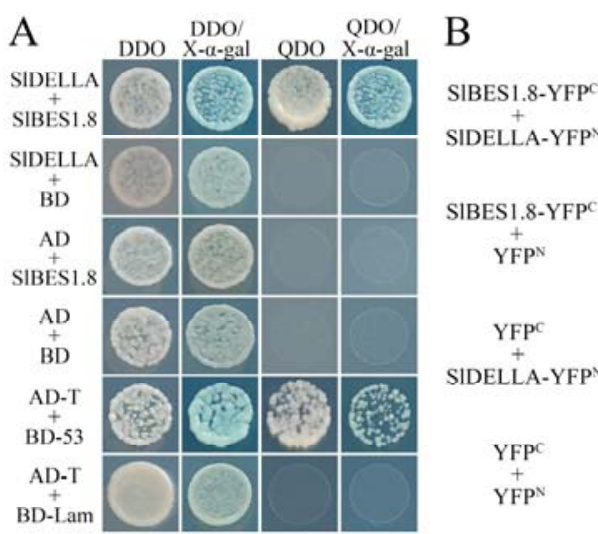

$\mathrm{D}$

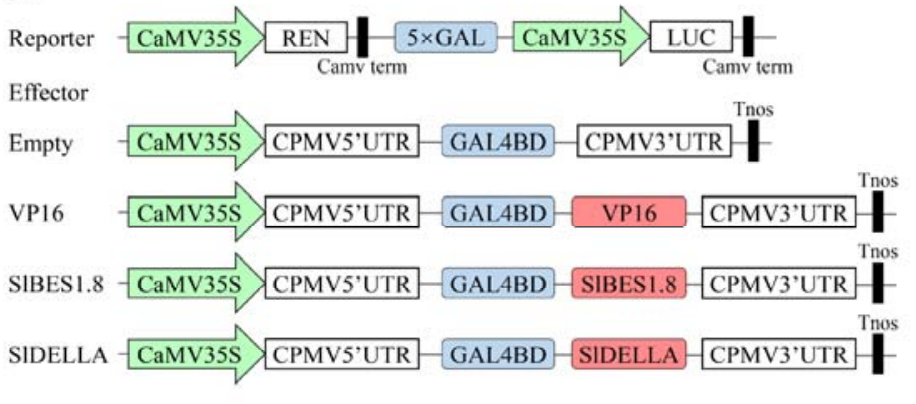

$\mathrm{F}$

\begin{tabular}{|c|c|c|c|}
\hline Biotin probe & + & + & \\
\hline GST-SIBES1.8 & + & + & \\
\hline ST-SIDELLA & & & \\
\hline
\end{tabular}

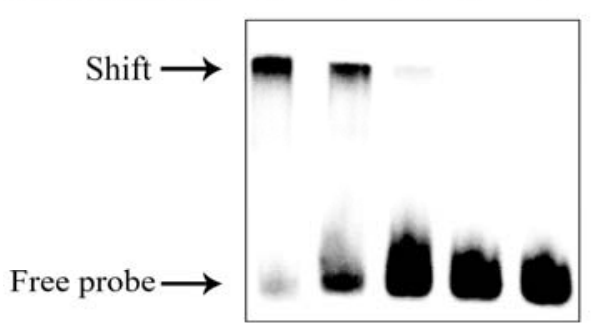

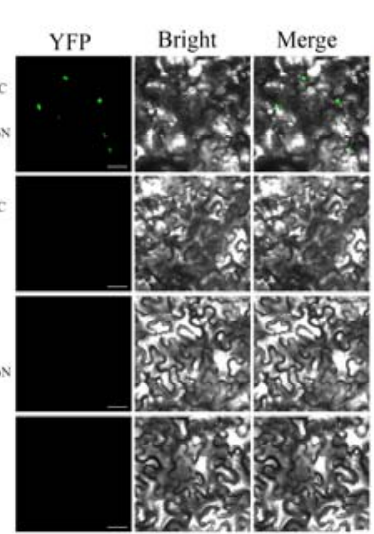

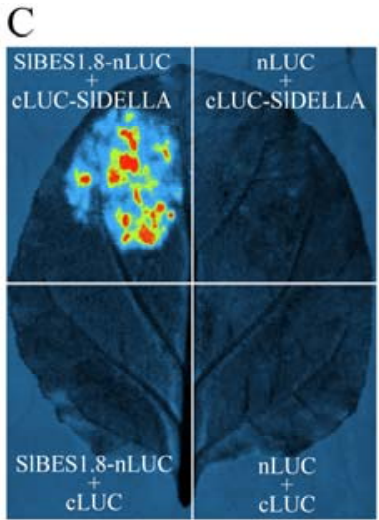

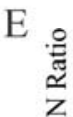

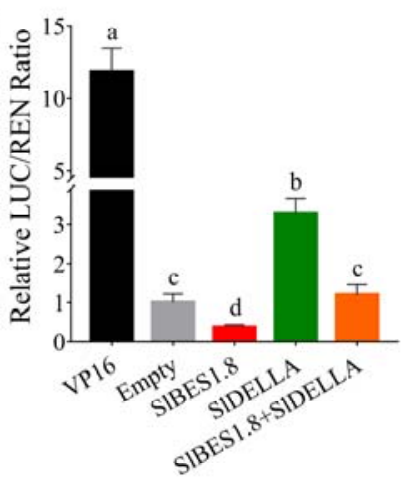

SIGIDIb-1

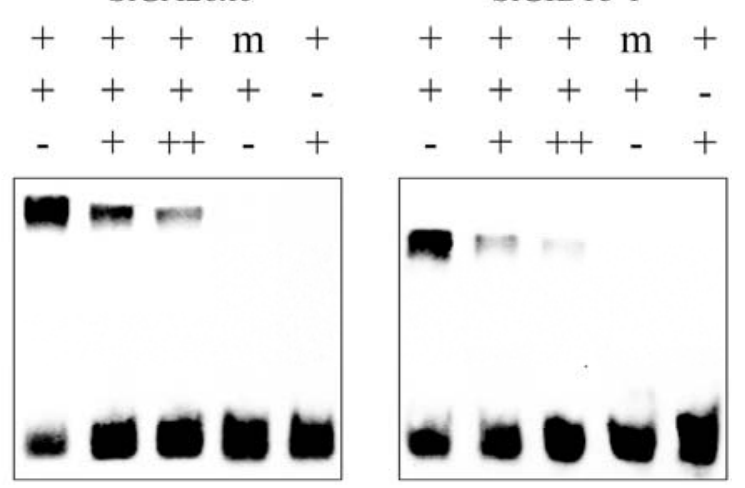

Fig. 7. SIDELLA interacts with SIBES1.8 and suppresses its transcriptional repression ability by inhibiting SIBES1.8-DNA binding. (A) Yeast two-hybrid $(\mathrm{Y} 2 \mathrm{H})$ assay for interaction between SIDELLA and SIBES1.8 proteins. AD-T plus BD-53 or BD-Lam were used as the positive and negative control respectively. DDO, SD/-Leu/-Trp medium; QDO, SD/-Ade/-His/-Leu/-Trp medium. (B) Bimolecular fluorescence complementation (BiFC) assay in tobacco leaves for interaction between SIBES1.8 and SIDELLA proteins. Bar $=50 \mu \mathrm{m}$. (C) Firefly luciferase complementation imaging (LCI) assay in tobacco leaves for interaction between SIBES1.8 and SIDELLA proteins. (D) Diagram of reporter and effectors used in dual-luciferase assays. SIDELLA and SIBES1.8 were fused with GAL4BD to generate the effector constructs. VP16 was used as the transcriptional activation control. (E) Suppression of SIDELLA on the transcriptional repression activity of SIBES1.8 detecting by GAL4-responsive reporter system in tobacco leaves. Reporter was 
co-expressed with SIBES1.8 or SIDELLA effector alone or together in tobacco leaves. The LUC/REN ratio of the group of empty effector was regarded as calibrator (set as 1). At least six biological replicates were used for each column. The significant differences are indicated with letters $(P<0.05$, one-way ANOVA test). (F) EMSA showing the inhibition of SIDELLA on the binding of SIBES1.8 to the promoters of SlGA2ox2, SlGA2ox6 and SlGID1b-1. Biotin probes were incubated with GST-SIBES1.8 alone or together with GST-SIDELLA protein. ++ indicates increased amount of GST-SIDELLA proteins; m, mutant biotin probe. 


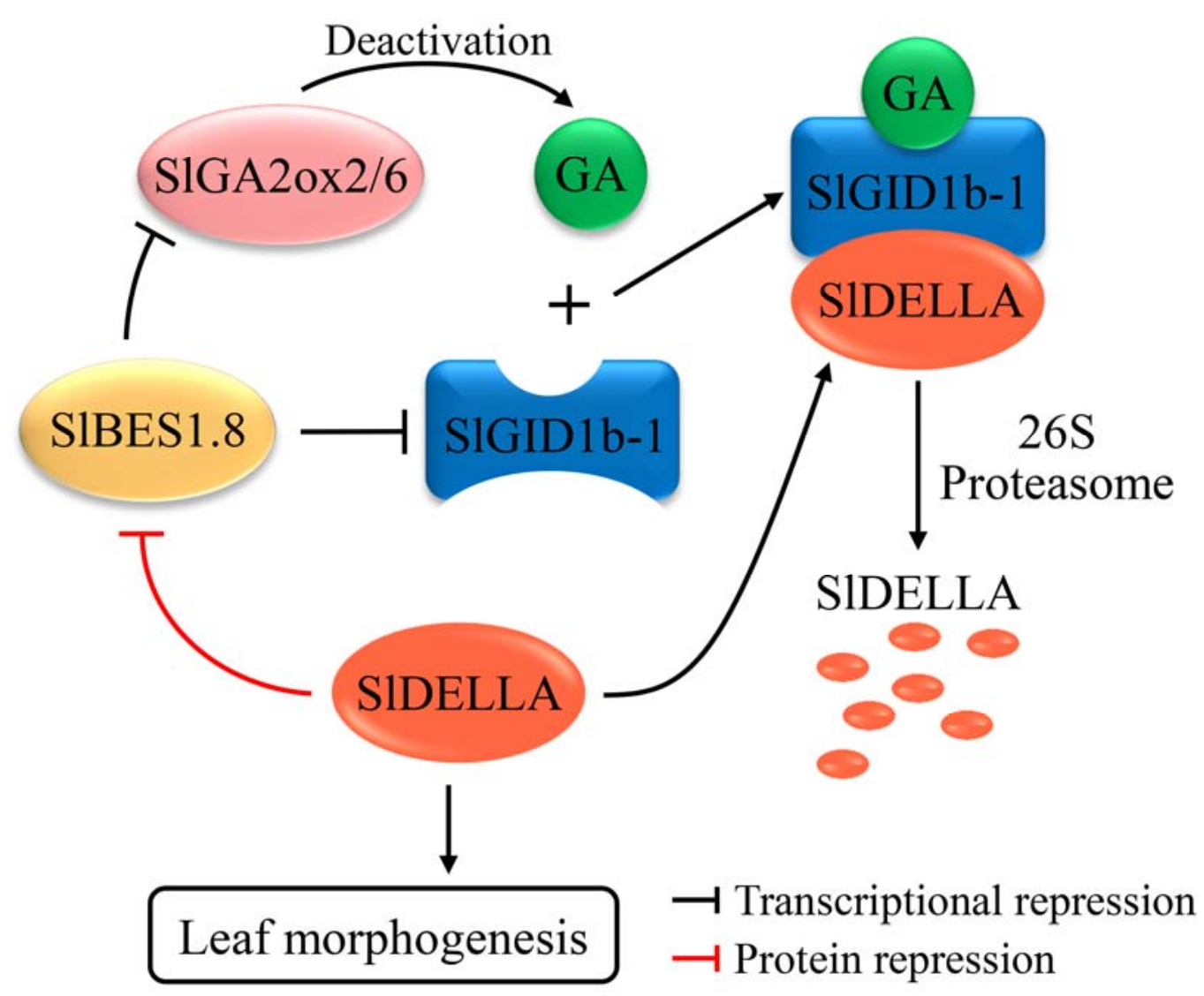

Fig. 8. Proposed model depicting the molecular mechanism of SIBES1.8 in the regulation of tomato leaf morphogenesis. SIBES1.8 represses the transcriptions of SlGA2ox2 and SlGA2ox6, which encode GA deactivation enzymes, to positively regulate bioactive GA contents and SIDELLA degradation. Meanwhile, SIBES1.8 also represses the expression of GA receptor, SlGIDIb-1, leading to a feedback regulation in SIDELLA degradation. On the other hand, SIDELLA physically interacts with SIBES1.8 and inhibits its transcriptional regulation ability. The degradation of SIDELLA releases its inhibition to SIBES1.8, which further promotes SIDELLA degradation. By such mediation both in GA level and signaling, SIBES1.8 greatly influences tomato leaf morphogenesis. 